\title{
2. COARSE FRACTION MINERALS OF SANDS IN CASCADIA MARGIN SEDIMENTS ${ }^{1}$
}

\author{
N.P. Chamov ${ }^{2}$ and I.O. Murdmaa ${ }^{3}$
}

\begin{abstract}
The coarse fraction $(0.05-0.1 \mathrm{~cm})$ of terrigenous sands cored in the rim of the deep-sea fan, in the slope basins, and in the accretionary wedge off the Washington-Oregon (Cascadia) Margin was obtained from all units at each site of Ocean Drilling Program Leg 146. The recognized mineral assemblages characterize two groups of sediments, which differ in age and physiographic setting. An amphibole-pyroxene-epidote-heavy mineral assemblage was defined in the Holocene to lower Pleistocene sediments from the deep-sea fan (Site 888), from the slope basins (Unit I at Sites 889/890), and from the anticlinal rise in the toe of the wedge (Site 891). An amphibole-epidote-heavy mineral assemblage was recognized in sands from the lower Pleistocene to upper Pliocene sediments of the accretionary wedge (Units II and III at Sites 889/890, and Site 892). An amphiboleepidote-heavy mineral assemblage is characterized by strong relative decrease in abundance of most heavy minerals against the background of a prominent increase in pyrite. This is accompanied by a significant increase in glauconite in the light fraction. Neither different sources nor sediment age alone is considered capable of significantly modifying the character of heavy mineral assemblages. An amphibole-epidote assemblage was defined at sites with anomalous fluid regimes and gas-hydrate occurrences, which seem to be important indicators of fluid influence on mineral assemblages. Chemically active fluids seem to have been responsible for intrastratal solution of heavy minerals, primarily, under burial conditions of chemically unstable pyroxene and amphibole. Changes in the character of heavy mineral assemblages have most probably resulted from strengthening of normal diagenesis by active fluids generated by tectonic overpressure in the accretionary wedge.
\end{abstract}

\section{INTRODUCTION}

The Oregon-Washington (Cascadia) Margin stretches along the western border of the North American continent for about $800 \mathrm{~km}$ from Vancouver Island, British Columbia in the North to Cape Blanco, Oregon in the South (Fig 1). Deep-sea Cascadia Basin is located seaward of the continental margin. Two large deep-sea fans (Nitinat and Astoria) are formed at the base of the Cascadia continental slope (Nelson et al., 1970; Carson, 1971).

The Cascadia Margin represents the convergence zone between the subducting oceanic Juan de Fuca Plate and the North American Plate (Fig. 1). The Juan de Fuca Plate has been converging toward the northeast and underthrusting the continent since Eocene. The modern convergence rate is approximately $42 \mathrm{~km} / \mathrm{m}$.y. (Riddihough, 1984; Duncan and Kulm, 1989; Demets et al., 1990). The rate of convergence is considered to remain stable for at least 10 m.y. (Hyndman et al., 1994).

The upper portion of the sedimentary section resting on the lithospheric plate has been accreted to the margin to form an accretionary wedge. The upward fluid migration caused by tectonic overpressure in the accretionary wedge is responsible for hydrocarbon locations near the base of the gas-hydrate stability field. This level is marked by the bottom-simulating reflectors (BSR) that are widespread at the Cascadia Margin (Hyndman, 1988; Hyndman et al., 1994; Westbrook, Carson, Musgrave, et al., 1994). Pore fluids have played an important role in determining the mechanical behavior of the accreted sediments and seem to have been responsible for alteration of mineral assemblages.

'Carson, B., Westbrook, G.K., Musgrave, R.J., and Suess, E. (Eds.), 1995. Proc. ODP, Sci. Results, 146 (Pt. 1): College Station, TX (Ocean Drilling Program).

${ }^{2}$ Geological Institute, Russian Academy of Sciences, Pyzhevsky Per. 7. Moscow 109017. Russia.

${ }^{3}$ Institute of Oceanology, Russian Academy of Sciences, ul. Krasikova 23, Moscow 117218 , Russia.
Ocean Drilling Program Leg 146 was directed toward investigation of tectonic dewatering in the accretionary wedge at the Cascadia continental margin (Westbrook, Carson, Musgrave, et al., 1994). Drilling at four sites penetrated sediments both off and within the accretionary wedge (Fig. 1). Site 888 provides a reference section for comparison with sites in the accretionary wedge on the continental margin.

Earlier, a group of sites were drilled to elucidate the geologic history of the Oregon continental margin and the adjacent Cascadia Basin in Leg 18 of the Deep Sea Drilling Project (DSDP) (Kulm, von Huene, et al., 1973). Drilling at these sites penetrated the Pleistocene to Pliocene sediments at the distal edge of the Astoria Fan (DSDP Site 174), at the Oregon lower continental slope (DSDP Site 175), and at the northern Oregon continental shelf (DSDP Site 176).

Terrigenous sands, silty sands, and sandy silts are common among sediments recovered at the Cascadia Margin in Leg 146. Coarse-fraction minerals are usually characteristic of certain source rock lithofacies and may be informative of postdepositional mineral alteration in accretionary wedges. We studied the mineral composition of the coarse fraction in sand from sediments both unaffected by accretionary tectonics (deep-sea fan and slope basins) and from those already incorporated into the accretionary wedge. Major goals of our study were to investigate the distribution of heavy and light minerals in stratigraphic sections, to compare the mineralogy of accreted and undeformed sedimentary sequences, and to evaluate the influence of fluids and accretionary tectonics on the mineral assemblages.

\section{METHODS}

The coarse fractions $(0.05-0.1 \mathrm{~mm})$ of sands were obtained by wet sieving from all units at each site of Leg 146. We selected 98 samples out of 393 samples for analysis. The heavy and light fractions were separated in bromoform $\left(2.9 \mathrm{~g} / \mathrm{cm}^{3}\right)$. We identified and counted 300 or more mineral grains per sample in each fraction using 
a polarizing microscope, then grouped some of them, and calculated percentage composition of each mineral (or mineral group) in both heavy and light fractions. From the heavy fraction, we grouped opaque minerals, rock fragments, and Fe-oxides as "opaques". Pyrite is shown separately to underline its variation in sites. Common hornblende and minor basaltic hornblende are counted together as "hornblende". Minerals of the epidote-zoisite group are counted as "epidote". From the light fraction, we counted quartz and rare fragments of chalcedony as "quartz". Clearly identified calcic, intermediate, and sodic plagioclases are counted as "plagioclases". Other feldspars with the refractive index $n<1.540$, together with trace amounts of microcline, are counted as "other feldspars". Results are presented in Tables 1 and 2.

\section{MINERALOGY OF SANDS} Sites off Vancouver

\section{Site 888}

Site 888 lies in the outer part of the Nitinat Fan, $7 \mathrm{~km}$ east of the toe of the accretionary wedge that forms the continental margin off Vancouver Island (Fig. 1). The holes at Site 888 penetrated into the top $600 \mathrm{~m}$ of the sedimentary section on the subducting oceanic plate. This section remains unaffected by accretionary tectonics.

Three lithostratigraphic units were recognized at Site 888 (Westbrook, Carson, Musgrave, et al., 1994). Holocene to upper Pleistocene Unit I (0-175.1 $\mathrm{m}$ below seafloor [mbsf]) includes interbedded clayey silts and fine- to medium-grained sands, with some thin beds containing pebbles, volcaniclastic fragments, and pieces of wood. Interval 175.1-193.0 mbsf (Cores 146-888B-19X to $-21 \mathrm{X}$ ) represents a transition between Units I and II. A gradual increase of massive sands is inferred from the progressive decrease of recovery and the dominance of sand in what was recovered in this interval. Geophysical data suggest the transitional nature of the lithologic change (Westbrook, Carson, Musgrave, et al., 1994). Upper Pleistocene Unit II (193.0-452.1 mbsf) is represented by thick ( $>1 \mathrm{~m})$ beds of massive, fine- to medium-grained poorly sorted sand with interbeds of clayey silt. Upper Pleistocene Unit III (452.1-566.9 mbsf) is composed of dark-gray, firm clayey silt and silt, finely laminated with thin interbeds of fine to coarse sand and gravel.

The three lithostratigraphic units are interpreted as submarine fan facies (Westbrook, Carson, Musgrave, et al., 1994). Facies identified in Unit II represent the middle fan environment. Facies recognized in Units I and III are characteristic of the outer fan environment. Turbidites in Unit III are more distal in character than in Unit I and appear to be more restricted to an abyssal plain environment. Two explanations were proposed in Leg 146 (Westbrook, Carson, Musgrave, et al., 1994) for the observed facies changes at Site 888. These changes were probably caused either by northward migration of the Nitinat Fan, or by travelling of Site's 888 location from the outer fan environment (Unit III) to the middle fan environment (the Nitinat Fan lobe, Unit II) and then to its present day position (Unit I).

The bulk of samples at Site 888 was obtained from Hole 888B; only one sample was taken from Hole $888 \mathrm{~A}$. We combined the data on these samples.

\section{Heavy Fraction}

The amphibole-pyroxene-epidote assemblage characterizes the heavy fraction and shows no significant variations through all units at Site 888 (Fig. 2A). Hornblende is most abundant (20\% to $40 \%$ ). It is relatively fresh and shows greenish-brown to bluish-green pleochroism. Two samples are relatively poor $(9 \%$ to $10 \%)$ in hornblende (Samples 146-888B-55X-CC, 0-6 cm, and 61X-01, 113-119 cm). Epidote and clinopyroxene are next in abundance (up to $20 \%$ through $25 \%$ ), with some prevalence of the former. Clinopyroxene is abundant in Samples 146-888B-11H-05, 126-130 cm, and 55X-CC, 0-6 $\mathrm{cm}$. Orthopyroxene is typically low (up to $10 \%$ ). Almost all py- roxenes are altered to varying degrees. Clinopyroxene and hornblende generally show an inverse relationship on the linear diagram.

Amphibole-epidote-pyroxene relationship data, shown on the triangular AEP diagram (Fig. 3A), plot in the central part of the triangle. Data from Unit II plot in the uppermost part of the field, reflecting some relative enrichment in amphibole. Data from Units I and II plot similarly, but data from Unit III demonstrate strongest dispersion and relative enrichment in epidote and pyroxenes. In general, data from all units at Site 888 represent the amphibole-pyroxene-epidote assemblage.

On the $(\mathrm{A}+\mathrm{Px}) \mathrm{EPr}$ triangular diagram (showing amphibole $+\mathrm{py}-$ roxene-epidote-pyrite relations), data from Units I and II plot very compactly along the $(\mathrm{A}+\mathrm{Px})-\mathrm{E}$ side (Fig. 4A). Data from Unit III show significant dispersion and generally stretch to the pyrite corner.

Magnetite occurs as discrete euhedral grains, fresh or abraded, and as aggregates. It is abundant (up to $10 \%$ through $17 \%$ ) in the middle to lower portion of Unit II (Samples 146-888B-30H-03, 74-78 $\mathrm{cm}$, through $-48 \mathrm{X}-\mathrm{CC}, 1-7 \mathrm{~cm}$ ) and is absent above and below this interval (Table 1). Garnet occurs in almost all sediments, but it is most significant (up to 10\%) throughout Unit II. Minerals of the actinolite-tremolite group are common (up to 5\%). Olivine, leucoxene, zircon, apatite, and sphene are present in minor (1\% to $3 \%$ ) amounts (Table 1). Rock fragments are represented by clay-chlorite aggregates, rarely silicified, and effusive rocks.

Some accessory minerals occur in trace amounts. These are chrome-spinel (Samples 146-888B-17H-01, 134-139 cm, and 28H02, 2-8 cm), spinel (Sample 146-888B-19X-01, 48-52 cm), anthophyllite (Sample 891B-50X-01, 37-40 cm), glaucophane (Samples 146-888B-27H-01, 12-27 cm; 28H-02, 37-51 cm; and 57X-01, 18 $24 \mathrm{~cm}$ ), sillimanite (Samples 146-888B-10H-02, 112-116 cm, and 17H-01, 134-139 cm), barite (Samples 146-888B-29H-06, 62-76 $\mathrm{cm} ; 54 \mathrm{X}-01,56-62 \mathrm{~cm}$, and $61 \mathrm{X}-01,113-119 \mathrm{~cm}$ ), aegyrine (Samples 146-888B-6H-04, 107-112 cm, through 7H-05, 94-99 cm; 29H$06,62-72 \mathrm{~cm}$, and $34 \mathrm{H}-05,19-25 \mathrm{~cm}$ ), anatase (in almost all samples above 259.92 mbsf and throughout Unit III), chloritoid (in almost all samples), and andalusite (Samples 146-888B-1H-04, 137-141 cm; $146-888 \mathrm{~B}-6 \mathrm{H}-04,107-112 \mathrm{~cm}$, to $29 \mathrm{H}-06,62-72 \mathrm{~cm}$, and $52 \mathrm{X}-\mathrm{CC}$, $2-8 \mathrm{~cm}$ to $61 \mathrm{X}-01,113-119 \mathrm{~cm}$ ).

\section{Light Fraction}

Quartz is the dominant component $(30 \%-50 \%)$ in the light fraction at Site 888 (Table 2). Quartz shows no significant variation through the site except for the lowest Sample 146-888B-61-01, 113$119 \mathrm{~cm}$, where it decreases up to $23 \%$. Plagioclase shows a gradual decrease in abundance from $20 \%$ in the upper portion of Unit I to $<5 \%$ in the lower portion of Unit II. Other constituents of the light fraction, occurring in minor proportions are, in order of decreasing abundance, micas, volcanic glass, and glauconite. None show any significant variation (never exceeding 10\%).

On the QFRf triangular diagram (representing quartz-feldsparrock fragments relations), data plot in the field slightly shifted from the center to the Q-Rf side and stretching in the rock fragments direction (Fig 5A). No evident trends in data distribution can be reported.

\section{Sites 889 and 890}

Sites 889 and 890 are located on the mid-continental slope off Vancouver Island (Fig. 1). Sediments at Sites 889 and 890 range in age from Holocene to late Pliocene. A hiatus at $88 \mathrm{mbsf}$ separates uppermost Quaternary sediments from lower Pleistocene through upper Pliocene sediments (Westbrook, Carson, Musgrave, et al., 1994).

Three lithostratigraphic units were recognized at Sites 889 and 890 (Westbrook, Carson, Musgrave, et al., 1994). Holocene to lower Pleistocene Unit I ( $0-128.0 \mathrm{mbsf})$ includes silty clays and clayey silts interbedded with very fine sand layers (1-8 cm thick). The most marked lithologic change is a downhole decrease both in frequency of sand layers and in their thickness. Lower Pleistocene to upper Pliocene Unit II (128.0-301.5 mbsf) consists of firm clayey silt and 
differs from Unit I by its lower abundance of sand and the reduced thickness of sand layers. The sediments of Unit II are firmer through compaction, are slightly cemented, and are noticeably more indurated. Indurated clayey silt is systematically fragmented into subangular pieces, 5 to $60 \mathrm{~mm}$ in diameter, which are supported by a clayey or silty matrix. The latter is either firm or soupy. Carbonate concretions, largely dolomitic, were observed throughout the section. Upper Pliocene Unit III (301.5-345.8 mbsf) is distinguished from Unit II only on the basis of significant increase in glauconite, with no other lithological or structural difference between these units.

Unit I comprises undeformed slope and slope-basin sediments that are hemipelagites, turbidites, and mass-flow deposits resting on the strongly deformed and brecciated sediments of the accretionary wedge (Units II and III). Units II and III are considered to represent abyssal-plain silts and clays that were postdepositionally fractured during accretion (Westbrook, Carson, Musgrave, et al., 1994). Based on known plate-motion rates, the age of the sediments recovered in Units II and III (Pliocene to early Pleistocene) suggests a paleogeographic position at the time of deposition about $100 \mathrm{~km}$ westward of the present position, in the Cascadia basin abyssal plain (Westbrook, Carson, Musgrave, et al., 1994).

Anomalously low temperatures observed in some cores, soupy disturbance in several layers, and the presence in such layers of fluids "diluted" by the melting of gas-hydrates provide evidence for gas-hydrate presence at this site. The large concentrations of methane found in the interval 130-247 mbsf could be indicative of saturation of pore water with methane, which probably had been released from hydrate that dissociated before being recovered on the ship (Westbrook, Carson, Musgrave, et al., 1994).

\section{Heavy Fraction}

All constituents of the amphibole-pyroxene-epidote assemblage show gradual decrease with depth at Site 889 (Fig. 2B). Hornblende decreases from about $39.23 \%$ at 23.38 mbsf (Sample 146-889A-1H$06,85-99 \mathrm{~cm}$ ) to less than $10 \%$ in Unit III. Except in the upper half of Unit I, clinopyroxene and hornblende demonstrate similar downhole distribution. Epidote decreases from $14 \%$ to $24 \%$ in Unit I to about $7 \%$ to $11 \%$ in Unit II, and to $3 \%$ to $6 \%$ in Unit III. Clinopyroxene decreases downhole from about $15 \%$ in Unit I, to less than $5 \%$ in Unit II, and to less than $1 \%$ to $2 \%$ in Unit III (Table 1). Orthopyroxene also gradually decreases from about $5 \%$ in Unit I to zero in Unit III. Pyroxenes are altered even in Unit I, and the degree of etching gradually increases with depth.

On the AEP triangular diagram, the data plot in a fan-shaped field that spreads from the center toward the side A-E (Fig. 3B). There is an evident trend within this field. Data from Unit I plot compactly in the central part of the triangle with some shift to the amphibole corner. Data from Unit II plot farther from the triangle center. Data from Unit III plot nearest to the A-E side and are dispersed along it. Based on the data distribution pattern, we can characterize data from Unit I at Site $889 / 890$ as the amphibole-pyroxene-epidote assemblage, and data from Unit III as the amphibole-epidote one. Data from Unit II represent somewhat of a transition between both assemblages.

The $(\mathrm{A}+\mathrm{Px})$ EPr triangular diagram also demonstrates a prominent trend in data distribution (Fig. 4B). Data from Holocene to lower Pleistocene Unit I plot along or near the $(\mathrm{A}+\mathrm{Px})-\mathrm{E}$ side tending to the $(\mathrm{A}+\mathrm{Px})$ corner, while data from Units II and III plot in a separate field near the pyrite corner (except Sample 146-889D-4N-01, 76-82 $\mathrm{cm}$, which plots in the field of Unit I). Within this separate field, data from the upper Pliocene Unit III plot closer to the pyrite corner than the data from the lower Pleistocene to upper Pliocene Unit II. This phenomenon (the deeper the sediment, the less amphibole, epidote, and pyroxene it contains) is most evident in Hole 889A, but it also could be observed in other holes at Site 889 , in spite of relatively high amounts of hornblende, pyroxene, and epidote registered in Samples 146-889B-14R-02, 12-15 cm, and 889D-4N-01, 76-82 cm. Contrary to the decrease of heavy minerals, pyrite strongly increases with depth (from about $1 \%$ to $30 \%$ in Unit I, from $55 \%$ to $70 \%$ in Unit II, and from $70 \%$ to $91 \%$ in Unit III).

Magnetite, actinolite-tremolite, and sphene occurring in minor amounts also show gradual decrease in abundance with depth (Table 1). The absence of olivine and leucoxene is also noticeable.

Minerals present in trace amounts are chrome spinel (Samples 146-890B-3H-04, 91-97 cm), sillimanite (Sample 146-890B-3H-02, 69-77 cm), barite (Samples 146-889A-1H-06, 85-99 cm; -2H-04, $60-65 \mathrm{~cm} ; 17 \mathrm{X}-02,55-65 \mathrm{~cm}$, and 20X-05, 47-52 cm), chloritoid (Sample 146-889A-31X-07, 13-17 cm), andanatase (Samples 146889A-4H-04, 49-55 cm; 1X-07, 13-17 cm; 889D-4N-01, 76-82 cm; $890 \mathrm{~B}-3 \mathrm{H}-02,69-77 \mathrm{~cm}$, and $5 \mathrm{H}-01,83-89 \mathrm{~cm}$ ).

\section{Light Fraction}

On the QFRf triangle (Fig. 5B) the data distribution is similar to that at Site 888 (Fig. 5A). The light fraction is dominated by quartz, which generally decreases downward from about $40 \%$ to $30 \%$ in Unit I to about $30 \%$ through $13 \%$ in Unit III (Fig. 6A).

Glauconite occurs sporadically in Unit I and is low in amount ( $0 \%$ to $4.21 \%$ ). It increases to $29.41 \%$ at 129.06 mbsf (Sample 146-889A$14 \mathrm{H}-\mathrm{CC}, 11-17 \mathrm{~cm}$ ) with a subsequent decrease with depth toward the lower portion of Unit II. Glauconite is extremely abundant (up to $70 \%$ ) in Unit III (Samples 146-889A-40X-01, 16-22 cm, through $44 \mathrm{X}-02,6-12 \mathrm{~cm})$. In general, quartz and glauconite show an inverse relationship (Fig. 6A).

Plagioclase and other feldspars are low in abundance (about 10\% each) and show no clear trends in their distribution. Volcanic glass is abundant $(45.79 \%)$ only at 245.74 mbsf (Sample 889A-31X-07, 13 $17 \mathrm{~cm}$ ). In general, volcanic glass and mica are common but low in amounts. Chlorite aggregates and chloritized rock fragments are common.

\section{Sites off Oregon}

\section{Site 891}

Site 891 lies on the westernmost ridge of the accretionary wedge at the foot of the Oregon continental margin (Fig. 1). The ridge is an anticlinally folded thrust sheet formed by movement along the frontal, landward-dipping fault that foots in the décollement beneath the wedge (Westbrook, Carson, Musgrave, et al., 1994).

Holocene Unit I at Site 891 consists of clayey silt, silt, and very fine to medium sand, with sporadic coarse sand, mud clasts, and shell fragments (Westbrook, Carson, Musgrave, et al., 1994). Allochthonous pebbles and carbonate concretions are distributed randomly throughout the section. Several cores below $200 \mathrm{mbsf}$ contain wood fragments. Three subunits were recognized within Unit I (Westbrook, et al., 1994). Subunit IA (0-198.2 mbsf) consists of clayey silt, interbedded with silt and fine to medium sand with faint parallel lamination in silts and sands. Tilted beds and convolute deformation sporadically occur. Subunit IB (198.2-383.9 mbsf) is composed of firm, fractured, unsorted clayey silt with minor amounts of sand. Sediments of the subunit are less sorted in comparison with adjacent subunits. Subunit IC (383.9-472.3 mbsf) includes clayey silt, sandy silt, and fine to medium sand. Sediments are less consolidated, less fractured, and better sorted than in Subunit ID. It is assumed (Westbrook, Carson, Musgrave, et al., 1994) that the sediments cored at Site 891 were not deposited in the present-day lower slope physiographic setting and represent deep-sea fan deposits uplifted and accreted to the lower slope of the Oregon margin.

\section{Heavy Fraction}

The bulk of samples was collected in Hole 891B and only one sample was obtained from Hole 891A. We combined these data.

On the linear diagram (Fig. 7) hornblende is evidently inversely related with clinopyroxene. The latter shows a gradual decrease in amount from $29.18 \%$ at 65.4 mbsf in Subunit IA (Sample 891B-8X- 
Table 1. Heavy fraction minerals of sands in Cascadia Margin sediments.

\begin{tabular}{|c|c|c|c|c|c|c|c|c|c|c|c|c|c|c|c|c|}
\hline $\begin{array}{l}\text { Core, section, } \\
\text { interval (cm) }\end{array}$ & $\begin{array}{l}\text { Depth } \\
\text { (mbsf) }\end{array}$ & Unit & Opq & Pyr & Mt & Ol & Lks & $\mathrm{Hb}$ & Ac- $\mathrm{Tr}$ & Cpx & Opx & Ep & $\mathrm{Gr}$ & $\mathrm{Zr}$ & Ap & Sph \\
\hline $\begin{array}{l}146-888 \mathrm{~A}- \\
\quad 1 \mathrm{H}-4,137-141\end{array}$ & 5.87 & I & 15.09 & 2.16 & 0 & 1.35 & 0.27 & 23.72 & 1.89 & 18.87 & 4.85 & 23.18 & 3.50 & 2.43 & 1.08 & 1.62 \\
\hline $\begin{array}{l}146-888 \mathrm{~B}- \\
6 \mathrm{H}-4,107-112\end{array}$ & 49.07 & & 18.14 & 2.21 & 0 & & & & & & & & & & & \\
\hline $7 \mathrm{H}-3,32-37$ & 56.34 & & $\begin{array}{l}15.29 \\
15.14\end{array}$ & 1.91 & 0 & 0.98 & $\begin{array}{l}0.25 \\
0.32\end{array}$ & $\begin{array}{l}24.26 \\
28.34\end{array}$ & $\begin{array}{l}1.96 \\
1.27\end{array}$ & $\begin{array}{l}14.95 \\
15.92\end{array}$ & $\begin{array}{l}4.41 \\
3.50\end{array}$ & $\begin{array}{l}27.21 \\
26.43\end{array}$ & 2.94 & 0.49 & 0.98 & 1.23 \\
\hline $7 \mathrm{H}-5,94-99$ & 59.96 & & 16.46 & 1.52 & 0 & 1.22 & 0.30 & 35.67 & 1.22 & 11.28 & 3.96 & $\begin{array}{l}26.43 \\
24.09\end{array}$ & $\begin{array}{l}3.18 \\
0.91\end{array}$ & $\begin{array}{l}0.64 \\
0.61\end{array}$ & $\begin{array}{l}0.64 \\
0.61\end{array}$ & $\begin{array}{l}1.59 \\
2.13\end{array}$ \\
\hline $9 \mathrm{H}-1,114-121$ & 73.14 & & 22.05 & 3.02 & 0 & 0.91 & 0.30 & 22.96 & 2.11 & 13.90 & 7.85 & 18.13 & 5.74 & 0.60 & 1.21 & 1.21 \\
\hline $10 \mathrm{H}-2,112-116$ & 84.12 & & 19.21 & 1.66 & 0 & 1.32 & 0.33 & 21.85 & 1.66 & 21.52 & 6.62 & 20.53 & 1.99 & 0.66 & 0.99 & 1.66 \\
\hline $11 \mathrm{H}-5,126-130$ & 98.16 & & 12.80 & 2.08 & 0 & 1.19 & 0.30 & 21.13 & 0.89 & 32.44 & 5.95 & 18.15 & 2.68 & 0.60 & 0.60 & 1.19 \\
\hline $17 \mathrm{H}-1,134-139$ & 147.94 & & 13.73 & 1.30 & 0 & 0.78 & 0.26 & 29.02 & 1.30 & 21.24 & 2.59 & 24.87 & 2.07 & 0.52 & 1.55 & 0.78 \\
\hline $19 X-1,48-52$ & 166.08 & & 13.31 & 2.55 & 0 & 1.70 & 0.28 & 32.58 & 4.53 & 14.45 & 2.55 & 22.95 & 1.98 & 1.42 & 0.85 & 0.85 \\
\hline $24 \mathrm{H}-3,93-107$ & 216.83 & II & 16.02 & 0.89 & 0 & 0.59 & 1.19 & 24.63 & 4.45 & 12.17 & 2.67 & 22.55 & 10.98 & 0.30 & 1.78 & 1.78 \\
\hline $25 \mathrm{H}-3,89-103$ & 221.29 & & 7.59 & 2.53 & 0 & 1.90 & 0.32 & 29.75 & 3.80 & 17.09 & 5.06 & 16.77 & 7.59 & 2.22 & 3.48 & 1.90 \\
\hline $27 \mathrm{H}-1,12-27$ & 234.32 & & 20.00 & 2.03 & 0 & 3.39 & 0.34 & 24.07 & 3.39 & 12.20 & 3.05 & 18.64 & 7.46 & 2.37 & 1.69 & 1.36 \\
\hline $27 \mathrm{H}-6,10-24$ & 241.80 & & 11.93 & 3.36 & 0 & 1.53 & 0.31 & 34.86 & 2.45 & 17.43 & 4.28 & 16.82 & 3.36 & 0.92 & 1.22 & 1.53 \\
\hline $28 \mathrm{H}-2,37-51$ & 245.18 & & 8.82 & 12.53 & 0 & 3.02 & 0.23 & 24.59 & 4.87 & 17.63 & 4.87 & 18.56 & 2.55 & 0.46 & 0.23 & 1.62 \\
\hline $29 \mathrm{H}-2,16-30$ & 253.46 & & 14.32 & 2.05 & 0 & 3.32 & 0 & 25.06 & 2.81 & 18.41 & 5.88 & 18.67 & 3.84 & 0.77 & 3.07 & 1.79 \\
\hline $29 \mathrm{H}-6,62-76$ & 259.92 & & 23.76 & 0.47 & 0 & 1.41 & 0.47 & 26.35 & 1.88 & 14.35 & 5.18 & 15.29 & 6.59 & 0.94 & 1.18 & 2.12 \\
\hline $30 \mathrm{H}-3,74-78$ & 264.54 & & 12.89 & 0 & 13.75 & 0.29 & 0.29 & 25.21 & 2.01 & 13.47 & 5.44 & 16.62 & 8.31 & 0.86 & 0.29 & 0.57 \\
\hline $31 \mathrm{H}-3,45-59$ & 271.29 & & 17.42 & 1.12 & 16.57 & 0.28 & 0.56 & 25.84 & 2.53 & 9.27 & 4.21 & 14.33 & 3.37 & 1.97 & 1.97 & 0.56 \\
\hline $33 \mathrm{H}-4,41-55$ & 285.70 & & 15.76 & 0.57 & 6.88 & 0.57 & 0 & 31.52 & 2.29 & 9.17 & 3.15 & 18.91 & 6.59 & 1.72 & 1.72 & 1.15 \\
\hline $34 \mathrm{H}-5,19-25$ & 298.51 & & 12.91 & 0 & 11.92 & 0 & 0 & 30.13 & 2.32 & 8.94 & 5.63 & 16.89 & 7.95 & 1.32 & 1.99 & 0 \\
\hline $35 \mathrm{H}-1,39-45$ & 300.89 & & 7.83 & 0 & 5.48 & 0.52 & 0.52 & 40.99 & 2.87 & 10.97 & 2.09 & 19.32 & 3.39 & 1.31 & 4.18 & 0.52 \\
\hline $35 \mathrm{H}-4,54-60$ & 305.54 & & 9.92 & 1.34 & 3.75 & 0.54 & 0.54 & 40.48 & 5.09 & 8.85 & 3.22 & 20.11 & 3.49 & 0.80 & 1.34 & 0.54 \\
\hline $36 \mathrm{H}-5,21-35$ & 314.44 & & 15.06 & 2.56 & 8.33 & 0.32 & 1.28 & 29.49 & 4.17 & 13.14 & 0.64 & 15.06 & 7.69 & 0.64 & 0.96 & 0.64 \\
\hline $37 \mathrm{H}-\mathrm{CC}, 9-15$ & 320.35 & & 16.39 & 5.74 & 9.56 & 0.27 & 0 & 20.49 & 1.09 & 18.31 & 3.55 & 16.67 & 5.74 & 0.55 & 0.55 & 1.09 \\
\hline $40 \mathrm{H}-2,30-37$ & 348.82 & & 15.00 & 1.88 & 7.50 & 0.63 & 0.31 & 25.94 & 2.19 & 15.63 & 2.81 & 18.75 & 4.38 & 0.63 & 2.19 & 2.19 \\
\hline $40 \mathrm{H}-6,76-90$ & 355.30 & & 12.09 & 2.36 & 11.80 & 0.88 & 0.29 & 28.02 & 2.06 & 11.21 & 2.95 & 19.47 & 5.90 & 0.59 & 1.77 & 0.59 \\
\hline $48 \mathrm{X}-\mathrm{CC}, 1-7$ & 423.51 & & 14,60 & 2.22 & 3.17 & 0 & 0.63 & 33.02 & 2.86 & 11.75 & 1.59 & 19.68 & 6.67 & 1.27 & 0 & 2.54 \\
\hline $52 \mathrm{X}-\mathrm{CC}, 2-8$ & 452.12 & III & 8.40 & 17.60 & 0 & 2.00 & 0.40 & 20.80 & 4.00 & 10.40 & 2.00 & 21.60 & 9.60 & 1.20 & 0.40 & 1.60 \\
\hline $54 \mathrm{X}-1,56-62$ & 469.96 & II & 7.14 & 2.81 & 0 & 0.51 & 0 & 29.59 & 3.06 & 20.41 & 4.08 & 25.00 & 1.79 & 0.77 & 3.32 & 1.53 \\
\hline $54 \mathrm{X}-1,66-72$ & 470.06 & & 10.25 & 9.19 & 0 & 1.41 & 0.35 & 21.55 & 2.12 & 22.97 & 7.07 & 15.90 & 4.59 & 1.06 & 2.12 & 1.41 \\
\hline $55 \mathrm{X}-\mathrm{CC}, 0-6$ & 478.40 & & 13.05 & 0.52 & 0 & 0.78 & 0.26 & 10.97 & 1.83 & 31.85 & 7.83 & 27.15 & 3.92 & 0.52 & 0.52 & 0.78 \\
\hline $57 \mathrm{X}-1,18-24$ & 496.38 & & 17.91 & 6.87 & 0 & 0.60 & 0.90 & 22.09 & 1.49 & 17.01 & 3.88 & 19.40 & 4.18 & 0.90 & 3.28 & 1.49 \\
\hline $61 X-1,113-119$ & 523.93 & & 9.52 & 44.81 & 0 & 0.22 & 0.65 & 9.74 & 1.30 & 9.96 & 3.46 & 16.88 & 1.08 & 0.65 & 0.65 & 1.08 \\
\hline $146-889 \mathrm{~A}-$ & & & & & & & & & & & & & & & & \\
\hline 1H-6, 85-99 & 23.38 & I & 16.08 & 0.64 & 1.61 & 0 & 0 & 39.23 & 1.93 & 14.47 & 2.89 & 18.65 & 2.57 & 0.64 & 0.96 & 0.32 \\
\hline $2 \mathrm{H}-4,60-65$ & 34.60 & & 22.63 & 1.68 & 5.31 & 0 & 0 & 29.05 & 1.96 & 16.20 & 2.79 & 17.04 & 1.96 & 0.56 & 0.28 & 0.56 \\
\hline $4 \mathrm{H}-4,49-55$ & 52.42 & & 9.32 & 5.65 & 0 & 0.28 & 0.28 & 28.25 & 2.54 & 17.23 & 4.80 & 24.29 & 1.69 & 0.85 & 2.26 & 2.54 \\
\hline $5 \mathrm{H}-6,84-87$ & 66.30 & & 4.84 & 31.29 & 0 & 0 & 0 & 20.65 & 3.23 & 15.48 & 2.90 & 16.45 & 1.94 & 1.94 & 0.97 & 0.32 \\
\hline $9 \mathrm{H}-\mathrm{CC}, 36-42$ & 103.84 & & 23.54 & 11.38 & 4.76 & 0 & 0.53 & 20.37 & 2.38 & 13.49 & 6.61 & 14.02 & 0.79 & 1.06 & 1.06 & 0 \\
\hline $14 \mathrm{H}-\mathrm{CC}, 11-17$ & 129.06 & ПI & 4.06 & 69.69 & 0 & 0 & 0 & 10.63 & 1.25 & 4.38 & 0.63 & 7.19 & 0.63 & 0.31 & 0.63 & 0.63 \\
\hline $17 \mathrm{X}-2,55-65$ & 131.31 & $\pi$ & 9.84 & 57.38 & 0.70 & 0 & 0 & 13.58 & 0.70 & 3.98 & 0.70 & 10.54 & 0.70 & 0.70 & 0.70 & 0.47 \\
\hline $20 \times-5,47-52$ & 164.82 & & 9.36 & 64.04 & 0 & 0 & 0 & 9.36 & 1.46 & 3.22 & 0.88 & 7.31 & 1.75 & 0.58 & 1,17 & 0.88 \\
\hline $31 \times-7,13-17$ & 245.74 & & 4.78 & 55.75 & 0 & 0 & 0.35 & 15.40 & 1.77 & 3.89 & 1.24 & 10.44 & 2.65 & 0.88 & 2.12 & 0.71 \\
\hline $40 \times-1,16-22$ & 301.66 & III & 7.08 & 75.08 & 0 & 0 & 0 & 7.69 & 0.92 & 1.85 & 0.31 & 3.69 & 0.31 & 2.46 & 0.62 & 0 \\
\hline $40 \times-2,69-75$ & 303.69 & & 3.21 & 79.29 & 0 & 0 & 0 & 8.57 & 1.07 & 0.71 & 0 & 5.00 & 0.71 & 0.71 & 0.36 & 0.36 \\
\hline $40 \times-3,10-16$ & 304.62 & & 3.48 & 87.11 & 0 & 0 & 0 & 5.92 & 0.35 & 0 & 0.35 & 2.09 & 0.70 & 0 & 0 & 0 \\
\hline $41 \times-1,6-12$ & 310.56 & & 8.46 & 71.79 & 0 & 0 & 0 & 6.90 & 0.94 & 2.19 & 0.31 & 5.96 & 0.31 & 1.88 & 1.25 & 0 \\
\hline $41 X-2,14-20$ & 312.14 & & 1.67 & 91.00 & 0 & 0 & 0 & 2.67 & 0.33 & 0.67 & 0 & 3.00 & 0.33 & 0.33 & 0 & 0 \\
\hline $44 X-2,6-12$ & 338.33 & & 1.55 & 83.59 & 0 & 0 & 0 & 5.57 & 0 & 0.93 & 0 & 5.88 & 1.55 & 0.31 . & 0.62 & 0 \\
\hline $\begin{array}{l}146-889 \mathrm{D}- \\
4 \mathrm{~N}-01,76-82\end{array}$ & 150.26 & II & 4.04 & 0.43 & 0 & 0.21 & 0 & 45.11 & 2.34 & 16.17 & 2.13 & 24.68 & 1.70 & 0.64 & 1.70 & 0.85 \\
\hline $\begin{array}{l}146-889 \mathrm{~B}- \\
14 \mathrm{R}-02,12-15\end{array}$ & 317.28 & III & 2.88 & 77.64 & 0 & 0 & 0 & 12.14 & 0.64 & 1.92 & 0.32 & 3.83 & 0.64 & 0 & 0 & 0 \\
\hline 146-890B- & & & & & & & & & & & & & & & & \\
\hline $3 \mathrm{H}-2,69-77$ & 18.93 & I & 6.18 & 20.22 & 0 & 0 & 0.28 & 30.62 & 2.81 & 16.01 & 2.25 & 15.45 & 2.53 & 0.56 & 1.69 & 1.40 \\
\hline $3 \mathrm{H}-4,39-44$ & 21.63 & & 14.86 & 1.76 & 3.78 & 0 & 0 & 30.98 & 3.02 & 13.60 & 2.77 & 16.62 & 8.06 & 1.51 & 2.02 & 1.01 \\
\hline $3 \mathrm{H}-4,91-97$ & 22.15 & & 5.80 & 6.76 & 0 & 0.97 & 0.24 & 28.02 & 2.42 & 14.49 & 3.14 & 25.85 & 5.31 & 0.72 & 4.35 & 1.93 \\
\hline $5 \mathrm{H}-1,83-89$ & 39.13 & & 6.12 & 3.99 & 0 & 0.53 & 0.53 & 34.31 & 2.39 & 17.02 & 3.72 & 22.07 & 2.13 & 1.06 & 3.46 & 2.66 \\
\hline $\begin{array}{l}146-891 \mathrm{~A}- \\
3 \mathrm{H}-1,111-125\end{array}$ & 8.41 & IA & 21.31 & 0.57 & 0 & 0.28 & 0.57 & 25.28 & 2.56 & 26.14 & 1.99 & 15.06 & 3.41 & 0.28 & 0.85 & 1.70 \\
\hline
\end{tabular}


Table 1 (continued).

\begin{tabular}{|c|c|c|c|c|c|c|c|c|c|c|c|c|c|c|c|c|}
\hline $\begin{array}{l}\text { Core, section, } \\
\text { interval }(\mathrm{cm})\end{array}$ & $\begin{array}{l}\text { Depth } \\
\text { (mbsf) }\end{array}$ & Unit & Opq & Pyr & Mt & Ol & Lks & $\mathrm{Hb}$ & Ac- $\mathrm{Tr}$ & $\mathrm{Cpx}$ & Opx & Ep & Gr & $\mathrm{Zr}$ & Ap & Sph \\
\hline \multicolumn{17}{|l|}{$146-891 \mathrm{~B}-$} \\
\hline $8 X-1,20-25$ & 65.40 & & 22.19 & 1.25 & 0 & 0.50 & 1.50 & 13.97 & 2.74 & 29.18 & 4.74 & 13.22 & 4.24 & 1.50 & 2.49 & 2.49 \\
\hline $11 X-1,6-12$ & 92.06 & & 28.57 & 5.52 & 0 & 1.95 & 0.32 & 12.99 & 1.95 & 24.68 & 4.55 & 11.36 & 2.92 & 0.65 & 2.27 & 2.27 \\
\hline $21 \mathrm{~N}-1,9-15$ & 171.69 & & 15.34 & 2.84 & 0 & 2.27 & 0.57 & 26.70 & 2.56 & 22.73 & 3.13 & 12.78 & 5.40 & 0.57 & 2.56 & 2.56 \\
\hline $23 \mathrm{X}-1,119-125$ & 181.69 & \multirow{4}{*}{ IB } & 15.22 & 7.76 & 0 & 0.31 & 0.31 & 22.36 & 3.42 & 18.01 & 2.17 & 17.70 & 5.90 & 0.31 & 1.55 & 4.97 \\
\hline $35 \mathrm{X}-1,124-130$ & 270.24 & & 18.18 & 2.27 & 7.47 & 0.32 & 0.65 & 22.40 & 0.97 & 16.56 & 2.92 & 18.51 & 4.22 & 1.62 & 3.25 & 0.65 \\
\hline $39 X-1,51-57$ & 295.61 & & 9.25 & 4.27 & 4.63 & 0 & 0.71 & 30.60 & 3.91 & 12.46 & 3.56 & 17.79 & 6.76 & 1.42 & 1.78 & 2.85 \\
\hline $40 \mathrm{X}-1,123-129$ & 305.23 & & 11.58 & 4.50 & 6.75 & 0 & 0 & 28.62 & 2.57 & 15.43 & 5.79 & 12.22 & 7.72 & 1.61 & 2.25 & 0.96 \\
\hline $41 X-2,97-103$ & 315.27 & \multirow{4}{*}{ IC } & 17.60 & 1.17 & 3.81 & 0.29 & 0 & 20.23 & 3.81 & $\begin{array}{l}18.48 \\
18.48\end{array}$ & 4.69 & 19.94 & 2.93 & 2.35 & 2.05 & 2.64 \\
\hline $50 \times-1,37-40$ & 393.17 & & 14.98 & $\begin{array}{l}1.17 \\
4.83\end{array}$ & 0.97 & 0.24 & 0 & 22.22 & 3.38 & $\begin{array}{l}18.40^{\circ} \\
18.8\end{array}$ & $\begin{array}{l}4.35 \\
4.35\end{array}$ & 16.91 & 6.52 & 2.66 & 2.90 & 1.21 \\
\hline $52 X-1,72-77$ & 411.22 & & $\begin{array}{l}14.98 \\
16.14\end{array}$ & 2.11 & 3.86 & $\begin{array}{l}0.24 \\
0\end{array}$ & 0.70 & 28.07 & 2.11 & $\begin{array}{l}18.84 \\
14.39\end{array}$ & $\begin{array}{l}4.40 \\
1.40\end{array}$ & 22.81 & $\begin{array}{l}0.52 \\
3.51\end{array}$ & $\begin{array}{l}2.00 \\
1.40\end{array}$ & 1.40 & 2.11 \\
\hline $58 \times-2,67-73$ & 465.12 & & 12.46 & 2.97 & 4.75 & 0.59 & 0.59 & 25.22 & 2.67 & 16.62 & 3.26 & 18.69 & 4.75 & 2.97 & 2.67 & 1.78 \\
\hline \multicolumn{17}{|l|}{$146-892 \mathrm{~A}-$} \\
\hline $4 X-2,14-20$ & 29.66 & IA & 5.21 & 85.42 & 1.04 & 0 & 0 & 1.04 & 0 & 0.69 & 0.69 & 4.17 & 1.04 & 0 & 0.35 & 0.35 \\
\hline $6 X-4,60-65$ & 44.12 & & 1.63 & 90.65 & 0 & 0 & 0 & 2.03 & 0.81 & 0.81 & 0.41 & 2.44 & 0.41 & 0 & 0 & 0.81 \\
\hline $14 \mathrm{X}-1,21-26$ & 106.71 & IB & 2.56 & 89.74 & 0 & 0 & 0 & 2.20 & 0 & 1.10 & 0.37 & 3.66 & 0.37 & 0 & 0 & 0 \\
\hline $15 \mathrm{X}-\mathrm{CC}, 8-14$ & 117.08 & & 2.53 & 77.62 & 0 & 0 & 0 & 10.47 & 0.72 & 3.61 & 0.72 & 3.25 & 0.72 & 0.36 & 0 & 0 \\
\hline $20 \mathrm{X}-2,76-82$ & 165.78 & & 8.67 & 65.33 & 3.41 & 0 & 0 & 8.05 & 1.55 & 3.10 & 0.62 & 5.26 & 0.93 & 1.24 & 1.24 & 0.62 \\
\hline $20 \mathrm{X}-\mathrm{CC}, 5-11$ & 167.17 & & 5.83 & 86.89 & 0 & 0 & 0 & 3.40 & 0.49 & 0.49 & 0.24 & 1.46 & 0.24 & 0.24 & 0.24 & 0.49 \\
\hline \multicolumn{17}{|l|}{ 146-892D- } \\
\hline $6 \mathrm{X}-3,53-59$ & 49.78 & IA & 13.85 & 84.62 & 0 & 0 & 0 & 0.77 & 0 & 0 & 0 & 0.38 & 0.38 & 0 & 0 & 0 \\
\hline $7 X-1,16-22$ & 54.16 & & 4.13 & 82.64 & 0 & 0.41 & 0 & 2.48 & 0.41 & 1.24 & 0.41 & 7.44 & 0.41 & 0 & 0.41 & 0 \\
\hline $7 X-6,30-36$ & 61.33 & & 2.65 & 85.88 & 0 & 0 & 0 & 6.47 & 0 & 0.59 & 0 & 2.35 & 0.29 & 0.29 & 0.88 & 0.59 \\
\hline $9 \mathrm{X}-3,67-72$ & 72.28 & IB & 3.04 & 84.30 & 0 & 0 & 0 & 6.58 & 0.76 & 0.51 & 0.25 & 2.78 & 0.25 & 0.25 & 0.76 & 0.51 \\
\hline 9 X-CC, 54-60 & 75.87 & & 4.97 & 69.34 & 0.55 & 0 & 0 & 9.94 & 2.21 & 3.59 & 0.83 & 6.63 & 1.66 & 0.28 & 0 & 0 \\
\hline $11 X-2,53-58$ & 111.53 & & 4.37 & 91.26 & $\begin{array}{l}0.30 \\
0\end{array}$ & 0 & 0 & 0.73 & 0.73 & 0.49 & 0.03 & $\begin{array}{l}0.03 \\
1.21\end{array}$ & 0.24 & 0.24 & 0.24 & 0.49 \\
\hline IIX-2, 138- 144 & 112.38 & & 11.63 & 71.32 & 0 & 0 & 0 & 3.88 & 1.94 & 1.94 & 0.78 & 6.20 & 1.16 & 0 & 0.39 & 0.78 \\
\hline $12 X-3,5-11$ & 122.05 & & 2.59 & 94.34 & 0 & 0 & 0 & 0.24 & 0.24 & 0.71 & 0.24 & 0.71 & 0.24 & 0.24 & 0.24 & 0.24 \\
\hline $12 X-3,91-97$ & 122.91 & & 8.83 & 82.86 & 0 & 0 & 0 & 3.38 & 0.78 & 0.52 & 0.26 & 2.34 & 0.26 & 0.26 & 0.26 & 0.26 \\
\hline $14 X-2,32-37$ & 139.62 & & 17.37 & 71.83 & 0 & 0 & 0 & 2.11 & 0.47 & 0.23 & 0 & 5.63 & 0.23 & 0.23 & $\begin{array}{l}1.17 \\
1.17\end{array}$ & 0.70 \\
\hline $15 \mathrm{X}-\mathrm{CC}, 12-18$ & 152.30 & & 19.15 & 75.53 & 0 & 0 & 0 & 0.71 & 0.53 & 0.53 & 0 & 2.30 & 0.53 & 0.18 & 0.35 & 0.18 \\
\hline $16 \mathrm{X}-4,52-57$ & 161.55 & & 13.98 & 81.45 & 0 & 0 & 0 & 0.54 & 0.27 & 0.54 & 0 & 2.15 & 0.27 & 0.27 & 0.27 & 0.27 \\
\hline $16 \times-4,128-132$ & 162.31 & & 5.68 & 85.79 & 0 & 0 & 0 & 0.71 & 0.53 & 1.07 & 0 & 4.80 & 0.18 & 0.53 & 0.36 & 0.36 \\
\hline $16 \times-5,16-21$ & $\begin{array}{l}102.31 \\
162.69\end{array}$ & & 8.02 & 83.02 & 0 & 0 & 0 & 0.95 & 1.72 & 0.19 & 0 & $\begin{array}{l}4.80 \\
3.63\end{array}$ & $\begin{array}{l}0.18 \\
1.34\end{array}$ & 0 & $\begin{array}{l}0.70 \\
0.76\end{array}$ & $\begin{array}{l}0.38 \\
0.38\end{array}$ \\
\hline $16 \mathrm{X}-5,94-100$ & 163.47 & & 2.76 & 80.34 & 0 & 0 & 0 & 7.93 & 2.76 & 0.69 & 0 & 5.17 & 0.34 & 0 & 0 & 0 \\
\hline $16 \mathrm{X}-\mathrm{CC}, 4-9$ & 164.19 & & 1.42 & 93.26 & 0 & 0 & 0 & 2.48 & 0.71 & 0 & 0 & 1.06 & 0.35 & 0 & 0.71 & 0 \\
\hline \multicolumn{17}{|l|}{$146-892 \mathrm{E}-$} \\
\hline $3 \mathrm{H}-1,10-15$ & 33.10 & IA & 1.42 & 92.17 & 0 & 0 & 0 & 0.36 & 0 & 0.36 & 0.36 & 4.27 & 0.71 & 0 & 0.36 & 0 \\
\hline $3 \mathrm{H}-1,80-85$ & 33.80 & & 6.30 & 88.98 & 0 & 0 & 0 & 1.97 & 0 & 0 & 0 & 2.36 & 0.39 & 0 & 0 & 0 \\
\hline $3 \mathrm{H}-3,10-15$ & 35.26 & & 25.81 & 56.13 & 0 & 0 & 0 & 4.52 & 0 & 4.52 & 0 & 7.74 & 0.65 & 0.65 & 0 & 0 \\
\hline $3 \mathrm{H}-3,35-40$ & 35.51 & & 2.22 & 68.33 & 0 & 0 & 0 & 7.22 & 0.56 & 2.78 & 0.56 & 11.11 & 1.11 & 5.00 & 1.11 & 0 \\
\hline $3 \mathrm{H}-3,130-135$ & 36.46 & & 6.99 & 89.15 & 0 & 0 & 0 & 0.37 & 0.18 & 0.37 & 0.18 & 1.84 & 0.37 & 0 & 0.37 & 0.18 \\
\hline $3 \mathrm{H}-4,13-19$ & 36.77 & & 6.75 & 84.42 & 0 & 0 & 0 & $\begin{array}{l}1.82 \\
.82\end{array}$ & 0.26 & 1.04 & 0.52 & $\begin{array}{l}3.04 \\
3.38\end{array}$ & 0.52 & 0.26 & 0.78 & 0.26 \\
\hline $3 \mathrm{H}-5,48-54$ & 38.59 & & 2.20 & $\begin{array}{l}87.42 \\
87.74\end{array}$ & 0 & 0 & 0 & 2.20 & 0.31 & $\begin{array}{l}1.04 \\
4.72\end{array}$ & 1.26 & 0 & 0.63 & 0.94 & 0 & 0.00 \\
\hline $3 \mathrm{H}-5,97-103$ & 39.08 & & 2.47 & 90.46 & $\begin{array}{l}0 \\
0\end{array}$ & 0 & 0 & 1.77 & 0.71 & $\begin{array}{l}4.72 \\
1.06\end{array}$ & 0.35 & 2.83 & $\begin{array}{l}0.03 \\
0\end{array}$ & 0.35 & 0 & 0 \\
\hline $3 \mathrm{H}-6,60-73$ & 40.23 & & 8.26 & 74.79 & 0 & 0 & 0 & 7.63 & 0.64 & 0.85 & 0 & 5.93 & 0 & 0.21 & 0.42 & 1.27 \\
\hline $3 \mathrm{H}-\mathrm{CC}, 12-20$ & 40.48 & & 9.09 & 78.71 & 0 & 0 & 0 & 2.44 & 1.11 & 0.89 & 0.22 & 5.76 & 0.22 & 0.67 & 0.44 & 0.44 \\
\hline
\end{tabular}

Notes: $\mathrm{Opq}=$ opaques; $\mathrm{Pyr}=$ pyrite; $\mathrm{Mt}=$ magnetite; $\mathrm{Ol}=$ olivine; $\mathrm{Lks}=$ leucoxene; $\mathrm{Hb}=$ hornblende; $\mathrm{Ac}-\mathrm{Tr}=$ actinolite-tremolite $(\mathrm{group}) ; \mathrm{Cpx}=$ clinopyroxene; Opx = orthopyroxene; $\mathrm{Ep}=$ epidote; $\mathrm{Gr}=$ garnet; $\mathrm{Zr}=$ zircon; $\mathrm{Ap}=$ apatite $\mathrm{Sph}=$ sphene. 
Table 2. Light fraction minerals of sands in Cascadia Margin sediments.

\begin{tabular}{|c|c|c|c|c|c|c|c|c|c|c|}
\hline $\begin{array}{l}\text { Core, section, } \\
\text { interval }(\mathrm{cm})\end{array}$ & $\begin{array}{l}\text { Depth } \\
\text { (mbsf) }\end{array}$ & Unit & Q & PI & $\mathrm{F}$ & M & Gic & $\mathrm{Vg}$ & $\mathrm{Rf}$ & Carb \\
\hline $\begin{array}{l}146-888 \mathrm{~A}- \\
\quad 1 \mathrm{H}-4,137-141\end{array}$ & 5.87 & I & 43.30 & 13.40 & 4.67 & 1.87 & 0 & 0 & 34.58 & 2.18 \\
\hline $\begin{array}{l}\text { 146-888B- } \\
6 \mathrm{H}-4,107-112 \\
7 \mathrm{H}-3,32-37 \\
7 \mathrm{H}-5,94-99 \\
9 \mathrm{H}-1,114-121 \\
10 \mathrm{H}-2,112-116 \\
11 \mathrm{H}-5,126-130 \\
17 \mathrm{H}-1,134-139 \\
19 \mathrm{X}-1,48-52 \\
24 \mathrm{H}-3,93-107 \\
25 \mathrm{H}-3,89-103 \\
27 \mathrm{H}-1,12-27 \\
27 \mathrm{H}-6,10-24 \\
28 \mathrm{H}-2,37-51 \\
29 \mathrm{H}-2,16-30 \\
29 \mathrm{H}-6,62-76 \\
30 \mathrm{H}-3,74-78 \\
31 \mathrm{H}-3,45-59 \\
33 \mathrm{H}-4,41-55 \\
34 \mathrm{H}-5,19-25 \\
35 \mathrm{H}-1,39-45 \\
35 \mathrm{H}-4,54-60 \\
36 \mathrm{H}-5,21-35 \\
37 \mathrm{H}-\mathrm{CC}, 9-15 \\
40 \mathrm{H}-2,30-37 \\
40 \mathrm{H}-6,76-90 \\
48 \mathrm{X}-\mathrm{CC}, 1-7 \\
52 \mathrm{X}-\mathrm{CC}, 2-8 \\
54 \mathrm{X}-1,56-62 \\
54 \mathrm{X}-1,66-72 \\
55 \mathrm{X}-\mathrm{CC}, 0-6 \\
57 \mathrm{X}-1,18-24 \\
61 \mathrm{X}-1,113-119\end{array}$ & $\begin{array}{r}49.07 \\
56.34 \\
59.96 \\
73.14 \\
84.12 \\
98.16 \\
147.94 \\
166.08 \\
216.83 \\
221.29 \\
234.32 \\
241.80 \\
245.18 \\
253.46 \\
259.92 \\
264.54 \\
271.29 \\
285.70 \\
298.51 \\
300.89 \\
305.54 \\
314.44 \\
320.35 \\
348.82 \\
355.30 \\
423.51 \\
452.12 \\
469.96 \\
470.06 \\
478.40 \\
496.38 \\
523.93\end{array}$ & III & $\begin{array}{l}41.70 \\
40.07 \\
47.66 \\
42.67 \\
36.05 \\
42.29 \\
50.32 \\
40.60 \\
46.07 \\
48.82 \\
34.80 \\
47.48 \\
40.16 \\
41.95 \\
40.59 \\
34.50 \\
35.15 \\
45.33 \\
36.24 \\
45.64 \\
39.29 \\
34.62 \\
40.56 \\
43.70 \\
45.02 \\
50.00 \\
45.83 \\
48.75 \\
32.37 \\
44.21 \\
40.18 \\
23.05\end{array}$ & $\begin{array}{r}22.39 \\
18.41 \\
17.45 \\
12.02 \\
14.16 \\
13.43 \\
111.08 \\
8.66 \\
14.29 \\
12.80 \\
16.12 \\
10.07 \\
9.84 \\
11.61 \\
10.56 \\
5.50 \\
4.60 \\
3.11 \\
6.99 \\
3.73 \\
4.59 \\
12.99 \\
3.21 \\
5.56 \\
3.90 \\
3.13 \\
5.45 \\
110.04 \\
4.56 \\
12.28 \\
18.93 \\
9.77\end{array}$ & $\begin{array}{l}6.56 \\
7.58 \\
6.38 \\
5.04 \\
8.15 \\
9.45 \\
0.13 \\
7.46 \\
5.36 \\
7.58 \\
6.23 \\
8.27 \\
7.09 \\
7.87 \\
4.95 \\
8.00 \\
5.02 \\
9.33 \\
8.30 \\
5.39 \\
8.67 \\
0.68 \\
8.03 \\
6.30 \\
6.49 \\
6.25 \\
9.29 \\
0.39 \\
4.15 \\
9.47 \\
0.42 \\
3.91\end{array}$ & $\begin{array}{r}2.70 \\
3.25 \\
1.70 \\
1.55 \\
1.72 \\
4.98 \\
1.90 \\
16.72 \\
3.57 \\
6.16 \\
4.03 \\
1.08 \\
4.72 \\
5.24 \\
4.95 \\
3.00 \\
2.51 \\
2.67 \\
1.31 \\
2.49 \\
10.71 \\
0.85 \\
2.01 \\
2.59 \\
1.30 \\
6.25 \\
6.73 \\
3.58 \\
2.49 \\
0.70 \\
1.49 \\
2.73\end{array}$ & $\begin{array}{l}0 \\
0 \\
0 \\
1.16 \\
0.86 \\
0 \\
0 \\
0 \\
0 \\
0 \\
0 \\
0 \\
0 \\
0 \\
0 \\
0 \\
0 \\
0 \\
0 \\
0 \\
0 \\
0 \\
0 \\
0 \\
0 \\
0 \\
0 \\
0 \\
0 \\
0 \\
0 \\
0 \\
0.83 \\
0.70 \\
0.60 \\
0\end{array}$ & $\begin{array}{l}0 \\
0.36 \\
0 \\
1.16 \\
0.43 \\
0.50 \\
0 \\
0 \\
0 \\
0 \\
0 \\
0 \\
0 \\
0.75 \\
0.66 \\
0.50 \\
0 \\
0 \\
0.44 \\
0.83 \\
0 \\
0.43 \\
0.40 \\
0.37 \\
1.73 \\
0 \\
0.64 \\
0 \\
0.83 \\
0 \\
0.60 \\
0\end{array}$ & $\begin{array}{l}23.18 \\
29.61 \\
24.68 \\
34.85 \\
36.48 \\
25.37 \\
22.14 \\
21.49 \\
25.35 \\
24.64 \\
35.16 \\
31.66 \\
35.43 \\
30.71 \\
34.66 \\
48.50 \\
52.72 \\
39.56 \\
46.72 \\
41.92 \\
36.74 \\
50.43 \\
45.79 \\
41.48 \\
41.56 \\
34.37 \\
26.59 \\
26.16 \\
54.36 \\
29.13 \\
36.60 \\
57.02\end{array}$ & $\begin{array}{l}3.47 \\
0.72 \\
2.13 \\
1.55 \\
2.15 \\
3.98 \\
4.43 \\
5.07 \\
5.36 \\
0 \\
3.66 \\
1.44 \\
2.76 \\
1.87 \\
3.63 \\
0 \\
0 \\
0 \\
0 \\
0 \\
0 \\
0 \\
0 \\
0 \\
0 \\
0 \\
5.47 \\
1.08 \\
0.41 \\
3.51 \\
1.18 \\
3.52\end{array}$ \\
\hline $\begin{array}{l}146-889 \mathrm{~A}- \\
1 \mathrm{H}-6,85-99 \\
2 \mathrm{H}-4,60-65 \\
4 \mathrm{H}-4,49-55 \\
5 \mathrm{H}-6,84-87 \\
9 \mathrm{H}-\mathrm{CC}, 36-42 \\
14 \mathrm{H}-\mathrm{CC}, 11-17 \\
17 \mathrm{X}-2,55-65 \\
20 \mathrm{X}-5,47-52 \\
31 \mathrm{X}-7,13-17 \\
40 \mathrm{X}-1,16-22 \\
40 \mathrm{X}-2,69-75 \\
40 \mathrm{X}-3,10-16 \\
41 \mathrm{X}-1,6-12 \\
41 \mathrm{X}-2,14-20 \\
44 \mathrm{X}-2,6-12\end{array}$ & $\begin{array}{r}23.38 \\
34.60 \\
52.42 \\
66.30 \\
103.84 \\
129.06 \\
131.31 \\
164.82 \\
245.74 \\
301.66 \\
303.69 \\
304.62 \\
310.56 \\
312.14 \\
338.33\end{array}$ & III & $\begin{array}{l}42.93 \\
37.55 \\
34.11 \\
31.31 \\
36.21 \\
35.29 \\
28.15 \\
33.33 \\
19.16 \\
15.41 \\
21.54 \\
21.72 \\
13.17 \\
14.34 \\
30.52\end{array}$ & $\begin{array}{r}8.78 \\
18.30 \\
16.36 \\
6.06 \\
13.32 \\
1.96 \\
5.93 \\
12.21 \\
13.55 \\
2.26 \\
3.85 \\
3.75 \\
2.06 \\
1.64 \\
2.91\end{array}$ & $\begin{array}{l}7.80 \\
0.92 \\
7.01 \\
6.57 \\
0.96 \\
8.82 \\
5.19 \\
8.45 \\
4.21 \\
5.26 \\
3.08 \\
4.87 \\
4.53 \\
2.87 \\
5.81\end{array}$ & $\begin{array}{l}0.98 \\
2.18 \\
2.80 \\
1.52 \\
0.66 \\
1.47 \\
2.59 \\
2.35 \\
2.80 \\
0.38 \\
0.38 \\
3.37 \\
0.41 \\
0 \\
0.29\end{array}$ & $\begin{array}{r}0.98 \\
1.31 \\
4.21 \\
0 \\
1.99 \\
29.41 \\
12.59 \\
7.51 \\
6.07 \\
54.51 \\
61.54 \\
28.09 \\
66.67 \\
70.90 \\
36.05\end{array}$ & $\begin{array}{l}0 \\
0.44 \\
1.87 \\
0.51 \\
0.33 \\
1.96 \\
4.81 \\
0.94 \\
45.79 \\
0 \\
0 \\
0.37 \\
0 \\
0 \\
0\end{array}$ & $\begin{array}{r}38.53 \\
39.30 \\
30.37 \\
54.03 \\
46.53 \\
21.09 \\
40.74 \\
34.27 \\
8.41 \\
22.18 \\
9.61 \\
37.83 \\
13.16 \\
10.25 \\
24.42\end{array}$ & $\begin{array}{l}0 \\
0 \\
3.27 \\
0 \\
0 \\
0 \\
0 \\
0.94 \\
0 \\
0 \\
0 \\
0 \\
0 \\
0 \\
0\end{array}$ \\
\hline $\begin{array}{l}\text { 146-889D- } \\
\quad 4 \mathrm{~N}-01,76-82\end{array}$ & 150.26 & II & 42.59 & 11.99 & 6.62 & 1.89 & 0.95 & 0.95 & 32.80 & 2.21 \\
\hline $\begin{array}{l}146-889 \mathrm{~B}- \\
14 \mathrm{R}-02,12-15\end{array}$ & 317.28 & III & 28.31 & 3.68 & 4.41 & 0.74 & 18.75 & 4.78 & 39.33 & 0 \\
\hline $\begin{array}{l}146-890 \mathrm{~B}- \\
3 \mathrm{H}-2,69-77 \\
3 \mathrm{H}-4,39-44 \\
3 \mathrm{H}-4,91-97 \\
5 \mathrm{H}-1,83-89\end{array}$ & $\begin{array}{l}18.93 \\
21.63 \\
22.15 \\
39.13\end{array}$ & 1 & $\begin{array}{l}47.42 \\
38.96 \\
44.22 \\
53.14\end{array}$ & $\begin{array}{r}10.65 \\
12.41 \\
12.87 \\
116.74\end{array}$ & $\begin{array}{l}4.81 \\
0.44 \\
5.94 \\
0.88\end{array}$ & $\begin{array}{l}1.03 \\
6.02 \\
6.93 \\
2.51\end{array}$ & $\begin{array}{l}2.06 \\
0 \\
1.65 \\
4.60\end{array}$ & $\begin{array}{l}0 \\
0 \\
0.66 \\
1.26\end{array}$ & $\begin{array}{l}31.28 \\
42.17 \\
25.42 \\
10.87\end{array}$ & $\begin{array}{l}2.75 \\
0 \\
2.31 \\
0\end{array}$ \\
\hline $\begin{array}{l}146-891 \mathrm{~A}- \\
3 \mathrm{H}-1,111-125\end{array}$ & 8.41 & IA & 49.33 & 12.08 & 9.73 & 2.35 & 0 & 2.01 & 20.14 & 4.36 \\
\hline $\begin{array}{l}146-891 \mathrm{~B}- \\
8 \mathrm{X}-1,20-25 \\
11 X-1,6-12 \\
21 \mathrm{~N}-01,9-15 \\
23 \mathrm{X}-1,119-125 \\
35 \mathrm{X}-1,124-130 \\
39 \mathrm{X}-1,51-57 \\
40 \mathrm{X}-1,123-129 \\
41 \mathrm{X}-2,97-103 \\
50 \mathrm{X}-1,37-40 \\
52 \mathrm{X}-1,72-77 \\
58 \mathrm{X}-2,67-73\end{array}$ & $\begin{array}{r}65.40 \\
92.06 \\
171.69 \\
181.69 \\
270.24 \\
295.61 \\
305.23 \\
315.27 \\
393.17 \\
411.22 \\
465.12 \\
\end{array}$ & IB & $\begin{array}{l}43.91 \\
39.44 \\
51.02 \\
51.00 \\
44.67 \\
51.94 \\
45.90 \\
44.84 \\
52.14 \\
47.96 \\
50.93\end{array}$ & $\begin{array}{r}116.67 \\
112.68 \\
13.27 \\
110.84 \\
14.92 \\
2.33 \\
16.34 \\
12.38 \\
22.50 \\
14.52 \\
16.48\end{array}$ & $\begin{array}{l}2.18 \\
0.56 \\
8.50 \\
3.25 \\
1.89 \\
7.36 \\
4.93 \\
6.27 \\
2.14 \\
1.76 \\
2.50\end{array}$ & $\begin{array}{l}9.94 \\
1.41 \\
5.44 \\
4.02 \\
4.51 \\
3.49 \\
3.73 \\
1.59 \\
2.50 \\
5.88 \\
6.94\end{array}$ & $\begin{array}{l}0 \\
0.70 \\
0.68 \\
0.80 \\
0 \\
0 \\
1.12 \\
0.40 \\
0 \\
0 \\
0\end{array}$ & $\begin{array}{l}1.28 \\
0.70 \\
0 \\
0 \\
2.46 \\
0.78 \\
1.87 \\
1.19 \\
0 \\
0 \\
1.39\end{array}$ & $\begin{array}{l}13.46 \\
30.64 \\
11.90 \\
13.66 \\
31.55 \\
34.10 \\
26.11 \\
33.33 \\
20.72 \\
29.88 \\
21.76\end{array}$ & $\begin{array}{l}2.56 \\
3.87 \\
9.18 \\
6.43 \\
0 \\
0 \\
0 \\
0 \\
0 \\
0 \\
0\end{array}$ \\
\hline $\begin{array}{l}146-892 \mathrm{~A}- \\
4 \mathrm{X}-2,14-20 \\
6 \mathrm{X}-4,60-65 \\
14 \mathrm{X}-1,21-26 \\
15 \mathrm{X}-\mathrm{CC}, 8-14 \\
20 \mathrm{X}-2,76-82 \\
20 \mathrm{X}-\mathrm{CC}, 5-11\end{array}$ & $\begin{array}{r}29.66 \\
44.12 \\
106.71 \\
117.08 \\
165.78 \\
167.17\end{array}$ & $\begin{array}{l}\text { IA } \\
\text { IB }\end{array}$ & $\begin{array}{r}24.83 \\
2.55 \\
13.83 \\
31.65 \\
28.47 \\
23.57\end{array}$ & $\begin{array}{l}3.40 \\
1.70 \\
0 \\
2.11 \\
5.24 \\
4.18\end{array}$ & $\begin{array}{l}8.16 \\
0.85 \\
1.19 \\
3.80 \\
6.74 \\
2.66\end{array}$ & $\begin{array}{l}0 \\
0 \\
2.37 \\
2.95 \\
1.12 \\
0.76\end{array}$ & $\begin{array}{l}19.05 \\
59.57 \\
7.51 \\
21.10 \\
26.97 \\
15.59\end{array}$ & $\begin{array}{l}2.38 \\
0.43 \\
0.40 \\
3.80 \\
4.87 \\
1.52\end{array}$ & $\begin{array}{l}42.18 \\
34.90 \\
74.70 \\
34.59 \\
26.59 \\
51.72\end{array}$ & $\begin{array}{l}0 \\
0 \\
0 \\
0 \\
0 \\
0\end{array}$ \\
\hline $\begin{array}{l}146-892 \mathrm{D}- \\
6 \mathrm{X}-3,53-59 \\
7 \mathrm{X}-1,16-22\end{array}$ & $\begin{array}{l}49.78 \\
54.16\end{array}$ & IA & $\begin{array}{r}6.25 \\
23.26\end{array}$ & $\begin{array}{l}1.56 \\
5.58\end{array}$ & $\begin{array}{l}1.56 \\
6.05\end{array}$ & $\begin{array}{l}0 \\
0\end{array}$ & $\begin{array}{l}72.66 \\
47.91\end{array}$ & $\begin{array}{l}0.39 \\
3.26\end{array}$ & $\begin{array}{l}17.58 \\
13.94\end{array}$ & $\begin{array}{l}0 \\
0\end{array}$ \\
\hline
\end{tabular}


Table 2 (continued).

\begin{tabular}{|c|c|c|c|c|c|c|c|c|c|c|}
\hline $\begin{array}{l}\text { Core, section, } \\
\text { interval }(\mathrm{cm})\end{array}$ & $\begin{array}{l}\text { Depth } \\
\text { (mbsf) }\end{array}$ & Unit & Q & Pl & $\mathrm{F}$ & M & Glc & $\mathrm{Vg}$ & Rf & Carb \\
\hline $7 X-6,30-36$ & 61.33 & \multirow{14}{*}{ IB } & 18.50 & 1.18 & 2.36 & 0 & 52.36 & 7.87 & 17.73 & 0 \\
\hline $9 X-3,67-72$ & 72.28 & & 28.01 & 4.56 & 5.21 & 0.33 & 25.08 & 4.89 & 31.92 & 0 \\
\hline $9 \mathrm{X}-\mathrm{CC}, 54-60$ & 75.87 & & 24.74 & 1.03 & 2.06 & 0 & 3.61 & 4.64 & 63.92 & 0 \\
\hline $11 X-2,53-58$ & 111.53 & & 32.82 & 111.79 & 2.31 & 2.56 & 7.18 & 9.23 & 24.11 & 0 \\
\hline $11 X-2,138-144$ & 112.38 & & 41.89 & 8.68 & 7.17 & 3.40 & 9.43 & 4.15 & 21.51 & 3.77 \\
\hline $12 X-3,5-11$ & 122.05 & & 21.25 & 9.16 & 1.83 & 1.83 & 26.37 & 1.47 & 38.10 & 0 \\
\hline $12 \times-3,91-97$ & 122.91 & & 15.93 & 2.21 & 4.42 & 3.10 & 7.08 & 7.08 & 60.18 & 0 \\
\hline $14 X-2,32-37$ & 139.62 & & 28.84 & 6.88 & 1.32 & 0.26 & 14.02 & 2.12 & 46.56 & 0 \\
\hline $15 \mathrm{X}-\mathrm{CC}, 12-18$ & 152.30 & & 15.75 & 4.76 & 3.66 & 2.56 & 26.74 & 4.76 & 41.77 & 0 \\
\hline $16 \times-4,52-57$ & 161.55 & & 19.93 & 7.69 & 2.80 & 1.40 & 48.60 & 0 & 18.18 & 1.40 \\
\hline $16 \times-4,128-132$ & 162.31 & & 17.46 & 5.95 & 3.57 & 1.19 & 26.98 & 0.79 & 43.27 & 0.79 \\
\hline $16 X-5,16-21$ & 162.69 & & 16.85 & 8.99 & 5.24 & 1.12 & 51.69 & 0 & 16.11 & 0 \\
\hline $16 \mathrm{X}-5,94-100$ & 163.47 & & 23.38 & 5.92 & 3.38 & 0.28 & 56.34 & 0 & 10.70 & 0 \\
\hline $16 \mathrm{X}-\mathrm{CC}, 4-9$ & 164.19 & & 22.49 & 6.83 & 3.61 & 2.01 & 56.63 & 0.80 & 7.63 & 0 \\
\hline \multicolumn{11}{|l|}{$146-892 \mathrm{E}-$} \\
\hline $3 \mathrm{H}-1,10-15$ & 33.10 & \multirow[t]{10}{*}{ IA } & 20.14 & 4.78 & 5.80 & 0.68 & 15.02 & 4.44 & 49.14 & 0 \\
\hline $3 \mathrm{H}-1,80-85$ & 33.80 & & 31.53 & 5.91 & 5.42 & 1.48 & 16.26 & 4.43 & 34.97 & 0 \\
\hline $3 \mathrm{H}-3,10-15$ & 35.26 & & 25.81 & 6.45 & 5.38 & 0 & 8.60 & 4.30 & 49.46 & 0 \\
\hline $3 \mathrm{H}-3,35-40$ & 35.51 & & 27.52 & 8.05 & 6.38 & 1.01 & 12.08 & 7.72 & 37.24 & 0 \\
\hline $3 \mathrm{H}-3,130-135$ & 36.46 & & 32.21 & 10.74 & 5.21 & 1.53 & 20.86 & 4.60 & 24.85 & 0 \\
\hline $3 \mathrm{H}-4,13-19$ & 36.77 & & 27.27 & 11.98 & 3.31 & 0.83 & 30.99 & 0 & 25.62 & 0 \\
\hline $3 \mathrm{H}-5,48-54$ & 38.59 & & 18.90 & 2.36 & 3.15 & 0 & 25.20 & 38.58 & 11.81 & 0 \\
\hline $3 \mathrm{H}-5,97-103$ & 39.08 & & 17.57 & 5.44 & 3.77 & 0.84 & 40.17 & 8.37 & 23.84 & 0 \\
\hline $3 \mathrm{H}-6,60-73$ & 40.23 & & 12.89 & 10.22 & 2.22 & 4.89 & 21.33 & 21.33 & 27.12 & 0 \\
\hline $3 \mathrm{H}-\mathrm{CC}, 12-20$ & 40.48 & & 37.74 & 11.02 & 4.41 & 1.93 & 18.46 & 3.86 & 22.58 & 0 \\
\hline
\end{tabular}

Notes: $\mathrm{Q}=$ quartz; $\mathrm{Pl}=$ clearly identified calcic, intermediate, and sodic plagioclases; $\mathrm{F}=$ other feldspars with refractive index $\mathrm{n}<1.540 ; \mathrm{M}=$ mica; $\mathrm{Gl} \mathrm{c}=$ glauconite; $\mathrm{Vg}=$ volcanic glass; $\mathrm{Rf}=$ rock fragments; $\mathrm{Carb}=$ carbonates.

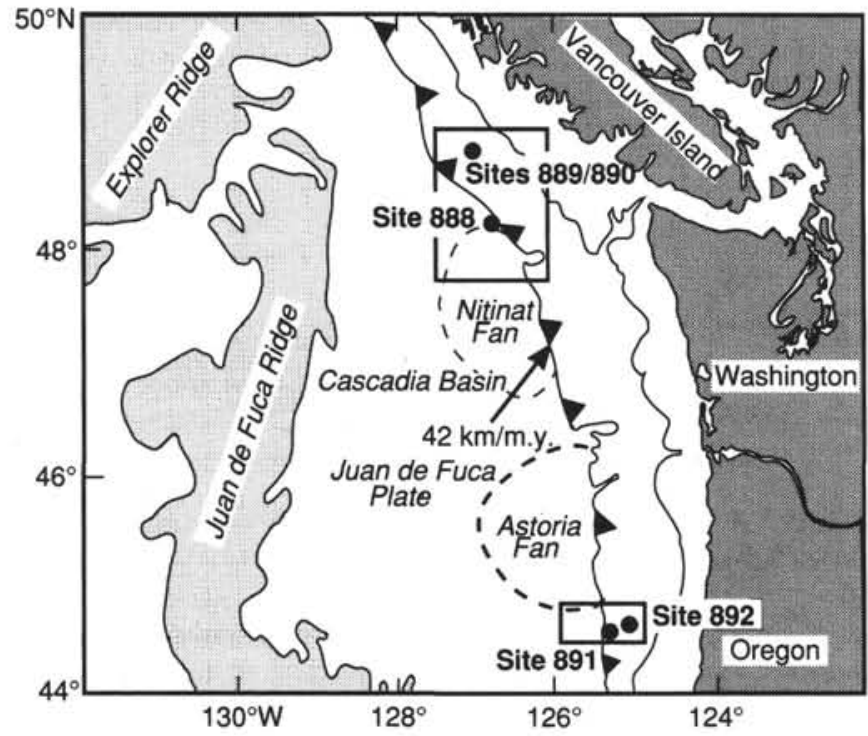

Figure 1. Location map of the Cascadia Margin, showing the convergent plate motion, major physiographic features in the Cascadia Basin (overlying the Juan de Fuca Plate), the position of the continental margin, and the position of drilling sites off Vancouver Island and Oregon (after Westbrook, Carson, Musgrave, et al., 1994).

01, 20-25 cm) to $12.46 \%$ at $296.61 \mathrm{mbsf}$ in Subunit IB (Sample $891 \mathrm{~B}-39 \mathrm{X}-01,51-57 \mathrm{~cm}$ ) with a subsequent increase in abundance up to about $17 \%$ at the boundary of Subunits IB and IC. Orthopyroxene is about $5 \%$ in abundance and shows no significant variations. Epidote also shows no clear trends in its distribution.

On the AEP triangular diagram, data plot very compactly in the central part of the triangle, except for the data from Samples 146891B-8X-01, 20-25 cm, and 11X-01, 6-12 cm (both from Subunit IA), which are shifted to the pyroxene corner (Fig. 3C). In general, data represent the amphibole-pyroxene-epidote assemblage.
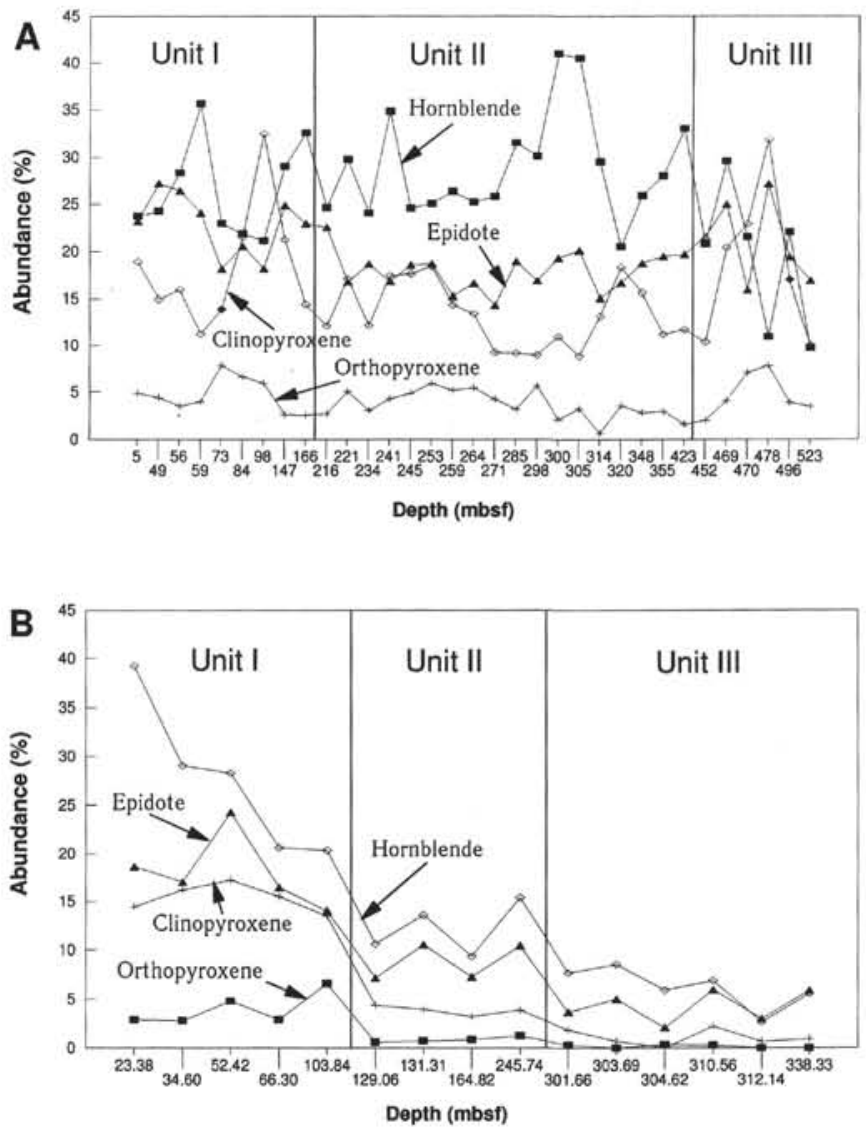

Figure 2. Downhole distribution of hornblende, pyroxenes, and epidote. A. Site 888 (Holes $888 \mathrm{~A}$ and $888 \mathrm{~B}$ ). B. Hole $889 \mathrm{~A}$. Depth values are rounded to the nearest integer. For complete values, see Table 1. 

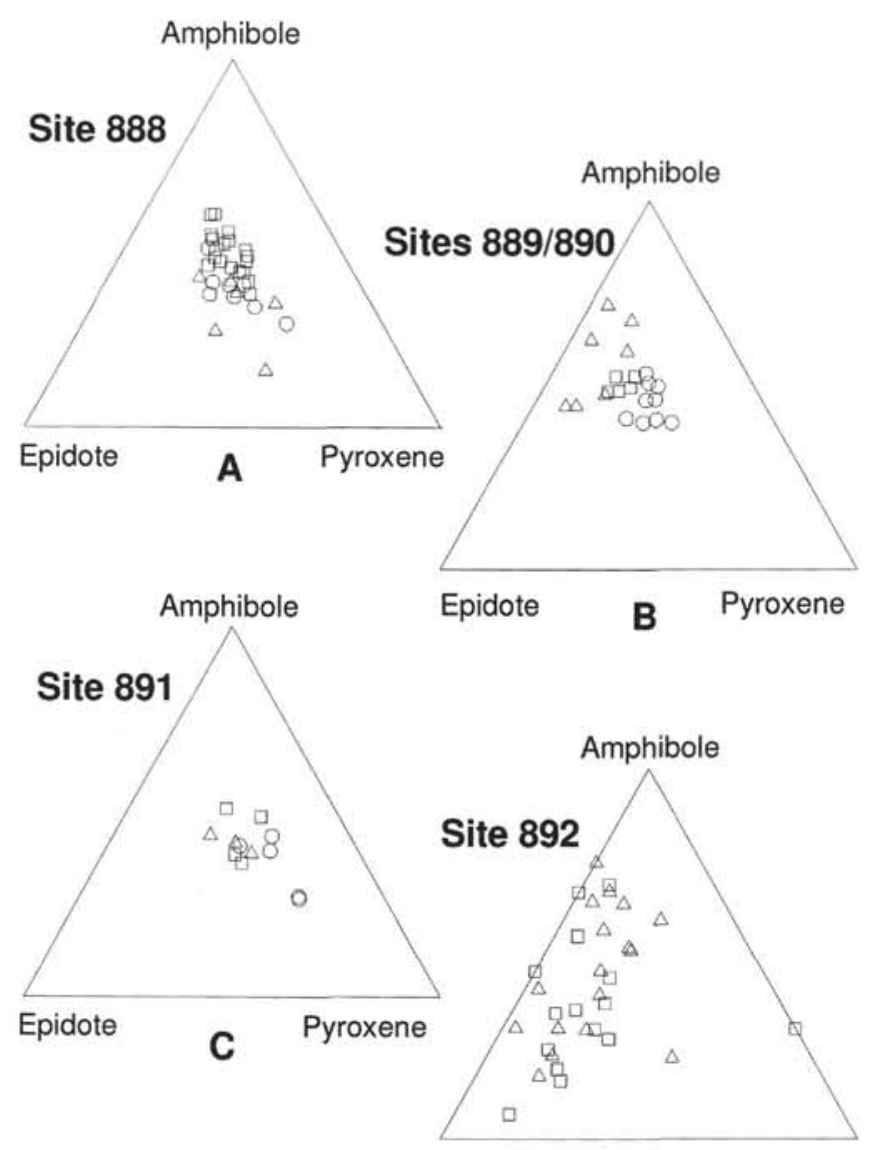

Epidote

D

Pyroxene

Figure 3. Triangular AEP diagrams showing amphibole-epidote-pyroxene distribution at Leg 146 sites. Hornblende and actinolite-tremolite are counted as "amphibole". A, B. Circle = Unit I, square = Unit II, triangle = Unit III. C. Circle $=$ Subunit IA, square $=$ subunit IB, triangle $=$ subunit IC. D. Square $=$ subunit IA, triangle $=$ subunit IB.

On the $(\mathrm{A}+\mathrm{Px})$ Epr diagram (Fig 4C), data from all subunits plot compactly along the $(\mathrm{A}+\mathrm{Px})-\mathrm{E}$ side tending to the $(\mathrm{A}+\mathrm{Px})$ corner. The data distribution pattern is similar to that of Site 888 Units I and II (Fig. 4A)

Olivine, leucoxene, zircon, apatite, garnet, and sphene occur in minor amounts. Minerals occurring in trace amounts are sillimanite (in almost all samples), aegyrine (Samples 146-891 A-3H-01, 111$125 \mathrm{~cm}$; 146-891B-8X-01, 20-25 cm; 23X-01, 119-125 cm; 40X-01, $123-129 \mathrm{~cm} ; 146-891 \mathrm{~B}-50 \mathrm{X}-01,37-40 \mathrm{~cm}$ through 146-891B-58X$02,67-73 \mathrm{~cm}$ ), chloritoid (in almost all samples except Subunit IC), tourmaline (in almost all samples), and andalusite (Samples 146891A-3H-01, $111-125 \mathrm{~cm}$; 146-892B-8X-01, 20-25 to $23 \mathrm{X}-01$, $119-125 \mathrm{~cm})$.

Each sample from Subunit IA contains trace amounts of anatase, barite, andalusite, rutile, and chlorite; they are absent in the lower subunits. No magnetite is registered in samples from Subunit IA, although it is abundant in Subunit IB (up to 8\%) and IC (up to 5\%).

\section{Light Fraction}

The triangular QFRf diagram (Fig. 5C) demonstrates notable differences in data distribution. Data from Subunit IA and IC plot in the field, stretching from the center toward the Q-F side, while the data from Subunit IB plot with an evident trend to the rock fragments corner.
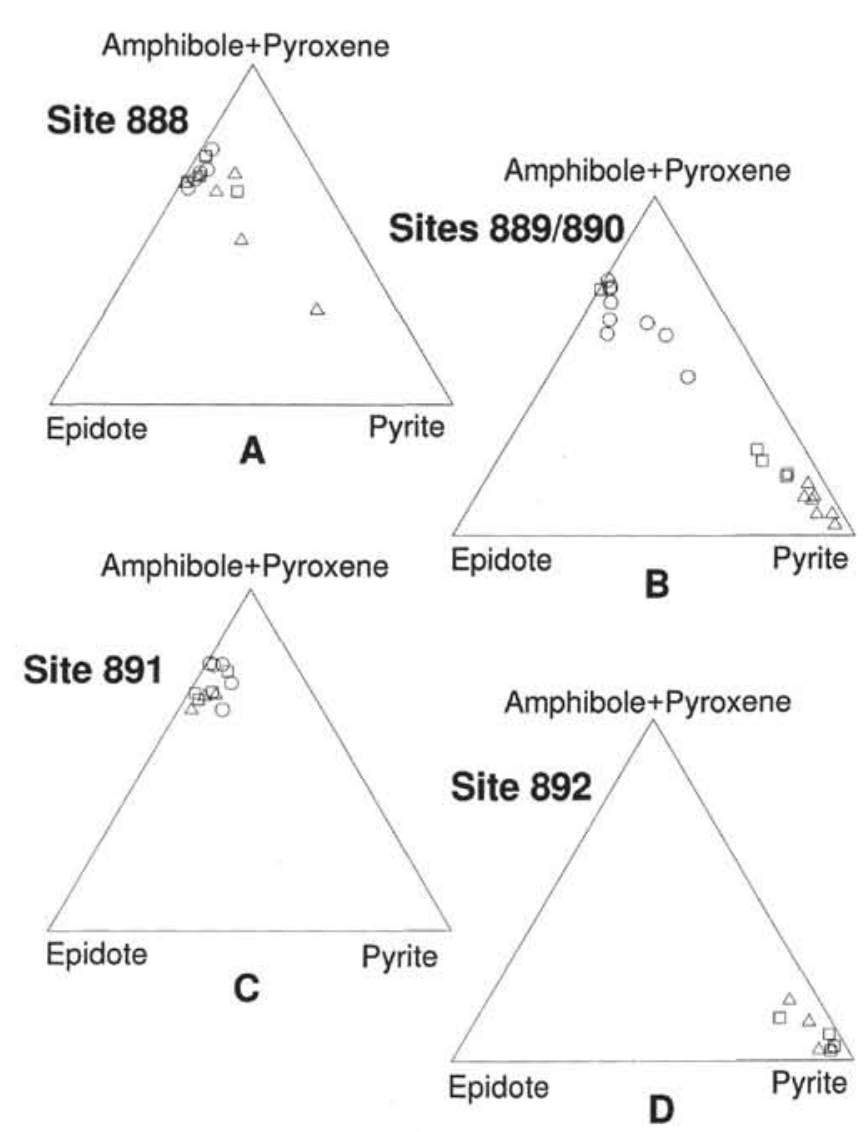

Figure 4. Triangular $(A+P x)$ EPr diagram showing amphibole + pyroxeneepidote-pyrite distribution at Leg 146 sites. To avoid having too many symbols that fall with in the same area, we drew every 2nd symbol for Unit I, every 4th symbol for Unit II (Site 888, Fig. 4A), every 4th symbol for Subunit IA, and every 5 th symbol for Subunit IB (Site 892, Fig. 4D). Simplified patterns in all cases adequately reflect the character of distribution and limits of the total data group. For symbols see Fig. 3 .

Quartz slightly varies (from about $40 \%$ to $50 \%$ ) throughout the entire site (Table 2). Plagioclase is low and gradually decreases with depth from about $10 \%$ to $16 \%$ at 65 mbsf to $2 \%$ through $6 \%$ in the lower subunits. Contrary to plagioclase, other feldspars demonstrate an increase in amount from about $10 \%$ in Subunit IA to about $20 \%$ at the top of Subunit IB with subsequent decrease in Subunit IC up to $10 \%$. Microcline is conspicuous in several samples.

Pale green, dark green, and almost colorless volcanic glass is a common constituent but low in abundance $(0.7 \%$ to $2.46 \%)$. Lithic fragments are fine-grained igneous rocks of basic to intermediate composition.

\section{Site 892}

Site 892 lies on the Oregon continental slope on the western flank of the second ridge within the accretionary wedge. Holes penetrated in the Pliocene section of the accretionary wedge and intersected both the bottom-simulating reflector (BSR) at $73 \mathrm{mbsf}$ and a hydrologically-active landward-dipping fault at 105 mbsf (Westbrook, Carson, Musgrave, et al., 1994). Because the lithologic character of lower? to upper Pliocene sediments was uniform, only one lithostratigraphic unit was identified (Westbrook, Carson, Musgrave, et al., 1994). Two subunits were recognized. Subunit IA (0-67.75 mbsf) contains a higher concentration of thin, terrigenous, and glauconitic sand layers 


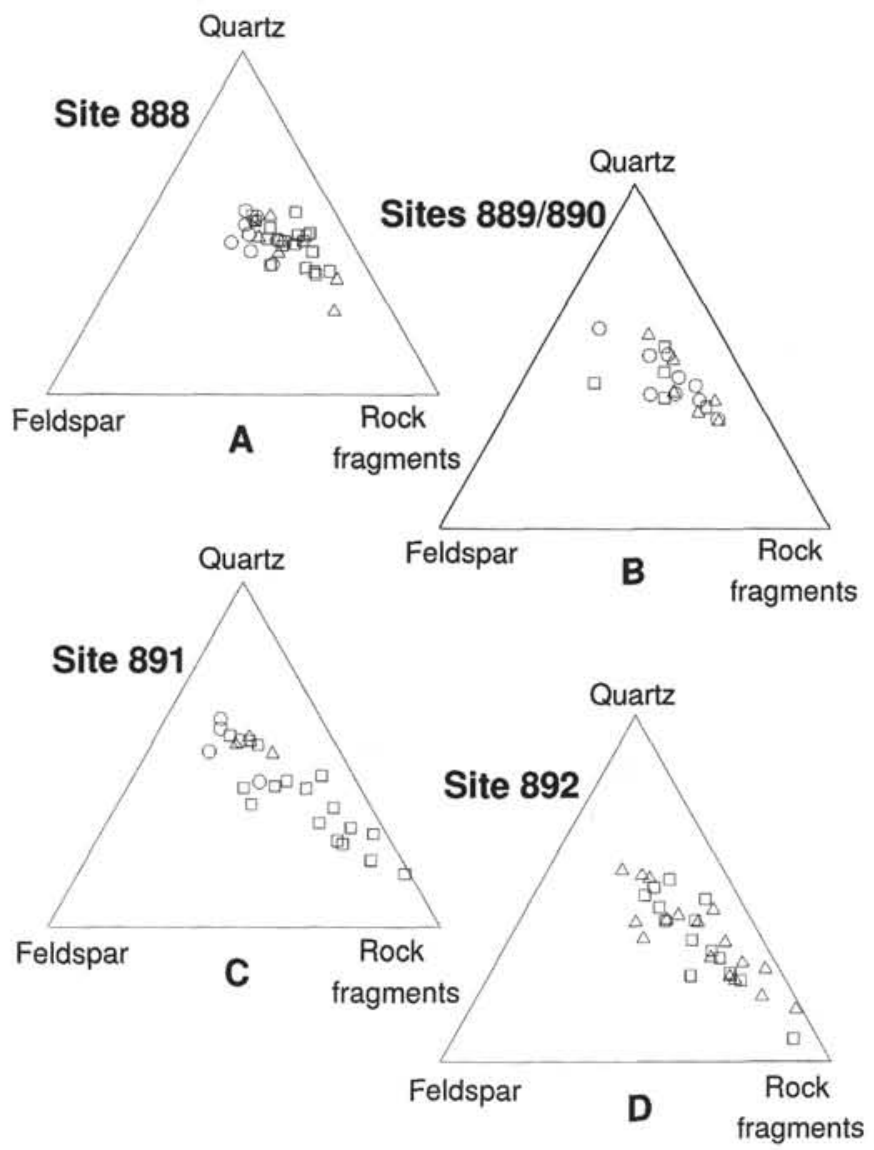

Figure 5. Triangular QFRf diagram showing quartz-feldspar-rock fragments distribution at Leg 146 sites. Clearly defined plagioclases and other feldspars with refractive index $n<1.540$ are counted as "feldspar". For symbols see Fig. 3.

and shows a downward increase of the mean grain size. Subunit IB contains fewer sandy layers and is intensely disturbed by fracturing and stratal disruptions at several intervals.

On the basis of preliminary observations, lithostratigraphic Unit I recovered at Site 892 was correlated (Westbrook, Carson, Musgrave, et al., 1994) with the basal portion of lithostratigraphic Unit II penetrated at DSDP Site 174 (Kulm et al., 1973). The latter, in turn, was correlated with the Pliocene to Pleistocene pre-Astoria Fan seismostratigraphic unit and was interpreted as produced by fine-grained, abyssal-plain sedimentation (Kulm, von Heune, et al., 1973). Thus, the sediments at Site 892 appear to be Pliocene abyssal-plain deposits postdepositionally incorporated into the accretionary wedge (Westbrook, Carson, Musgrave, et al., 1994).

Gas-hydrates were recovered as macroscopic crystals, pellets, and aggregates from the upper $19 \mathrm{~m}$ of sediments at Site 892. Gas-hydrates are also supposed to be present as disseminated deposits to 75 mbsf (Westbrook, Carson, Musgrave, et al., 1994). A hydrologicallyactive, landward-dipping fault was intersected at this site.

\section{Heavy Fraction}

Data from Site 892 obviously represent the amphibole-epidote assemblage. On the AEP triangular diagram (Fig 3D), data from both subunits plot along or directly on the A-E side. This data distribution pattern is very similar to that from Site $889 / 890$ Units II and III sediments (Fig. 3B), although it reflects relative enrichment in epidote.
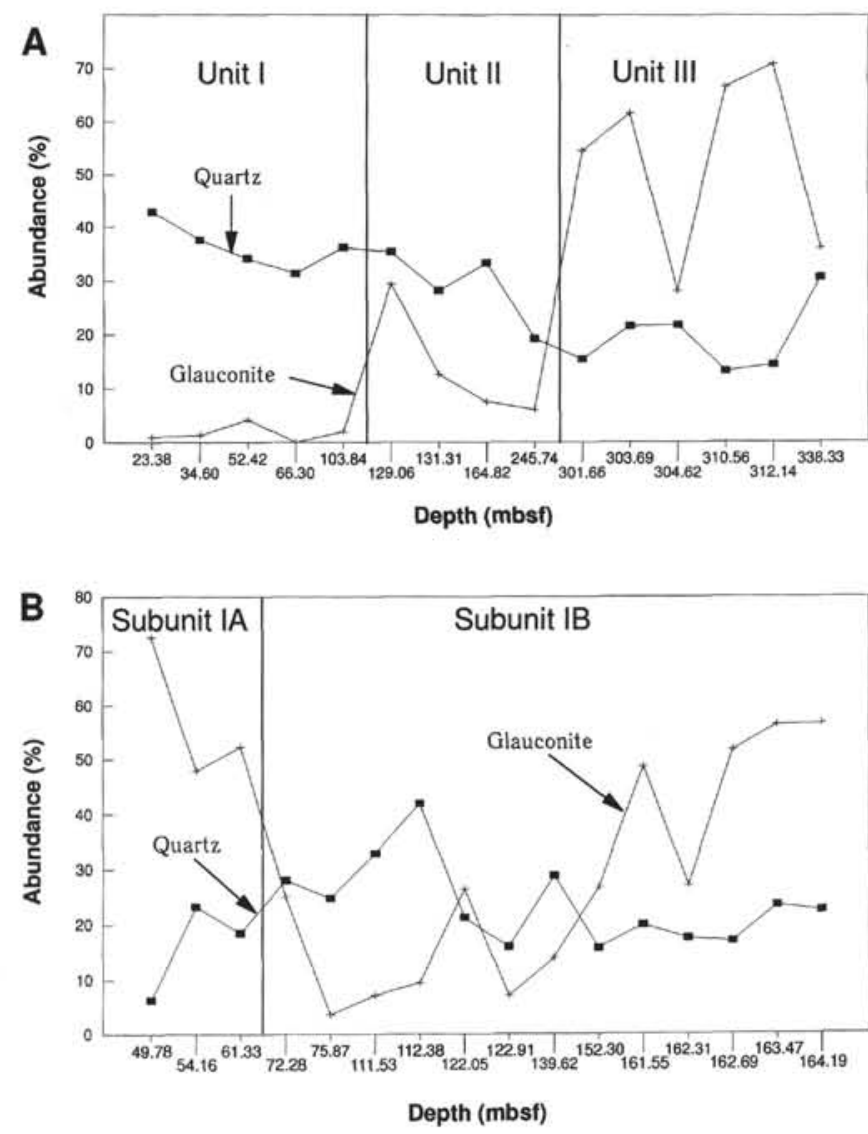

Figure 6. Downhole distribution of quartz and glauconite. A. Hole 889A. B. Hole 892D.

The data from Samples 146-892D-12X-03, 5-11 cm, and 146-892E$3 \mathrm{H}-05,48-54 \mathrm{~cm}$, provide some exceptions from this pattern.

On the (A + Px)EPr triangular diagram (Fig. 4D) data plot in the pyrite corner, reflecting strong decrease in amphibole, pyroxene, and epidote (to $1 \%$ through $5 \%$ ) against the background of increase in pyrite (up to $90 \%$ ). This data seem to resemble data from Units II and III at Site 889.

Other mineral constituents show also low abundance. Garnet, zircon, apatite, and sphene, known as minor in other sites, occur in trace amounts, if at all. Magnetite, olivine, and leucoxene are almost absent. Some minerals are present in trace amounts. These are glaucophane (Sample 146-892E-3H-CC, 12-20 cm), sillimanite (Sample 146-892D-11X-02, 138-144 cm), barite (Samples 146-892A-6X-04, $60-65 \mathrm{~cm} ; 146-892 \mathrm{D}-6 \mathrm{X}-03,53-59 \mathrm{~cm}$, and 146-892E-3H-03, 35$40 \mathrm{~cm}$ ), chloritoid (Samples 146-892D-11X-02,138-144 cm and 16X-CC, 4-9 cm), and anatase (Samples 146-892E-3H-04, 13-19 cm and 146-892D-16X-04, 128-132 cm).

\section{Light Fraction}

On the QFRf triangular diagram (Fig. 5D), data from both subunits plot in the elongated field from the center to the rock fragments corner with no evident inner trends. The pattern is generally similar to that of data from Site 891 Subunit IB (Fig. 5C).

Glauconite is extremely abundant in Subunit IA and in the lower portion of Subunit IB, showing a definite inverse relationship with quartz (Fig 6B). The glauconite pellets are dark green and of fine- to 


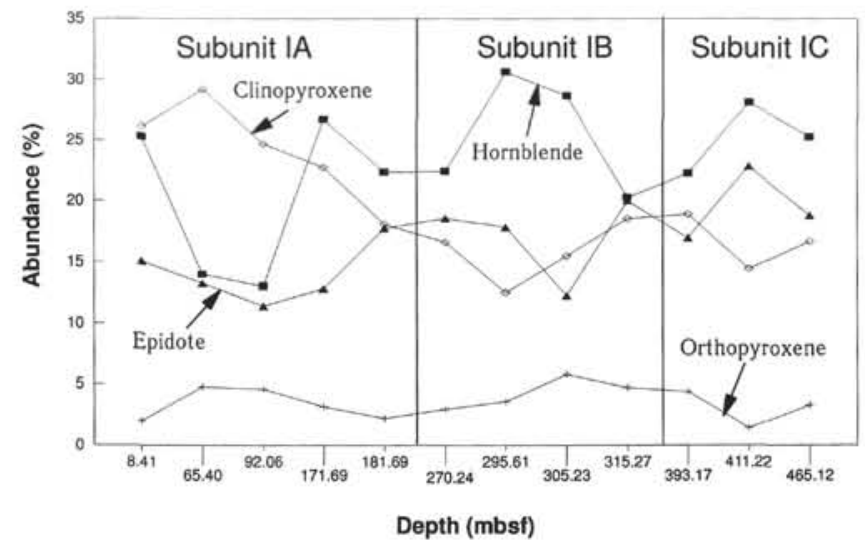

Figure 7. Downhole distribution of hornblende, pyroxene, and epidote at Site 891 (Holes 891A and 891B).

medium-grain size, commonly with rims of pale green clay and pyrite. Volcanic glass seems to be more widespread in both sites off Oregon, compared to those off Vancouver Island.

\section{DISCUSSION AND CONCLUSION}

The obtained data demonstrate significant contrasts in mineral assemblages in sand from sediments recovered at the Washington-Oregon (Cascadia) Margin. We defined amphibole pyroxene epidoteheavy mineral assemblage in the Holocene to lower Pleistocene sediments from the deep-sea fan (Site 888), from the slope basins (Unit I at Sites 889/890), and from the anticlinal rise in the toe of the accretionary wedge (Site 891). Amphibole epidote-heavy mineral assemblage was recognized in sands from the lower Pleistocene to upper Pliocene sediments of the accretionary wedge (Units II and III at Sites $889 / 890$, and Site 892). Thus, two mineral assemblages characterize two groups of sediments, which differ in age and physiographic setting.

Different sources, age, accretionary tectonics, and fluid regimes may have resulted in the formation of these two assemblages. The amphibole pyroxene epidote-heavy mineral assemblage in the Holocene to lower Pleistocene sands was most probably formed in or near to the present physiographic setting. Amphibole-rich (and especially hornblende-rich) land masses of Vancouver Island and western British Columbia as well as amphibole pyroxene-rich Oregon's and Washington's Cascade Range seem to have been the main source for these assemblages (Easterbrook, 1963; Wiese, 1969; Kulm et al., 1968; Whetten et al., 1969; Duncan and Kulm, 1970; Carson, 1971; Sheidegger et al., 1971; Carlson and Nelson 1987). Immature pyroxene amphibole-heavy mineral assemblage was also recognized in the Pleistocene Astoria Fan sediments recovered during DSDP Leg 18 (Scheidegger et al., 1973).

The amphibole epidote-heavy mineral assemblage in the lower Pleistocene to upper Pliocene sands probably formed in the basinplain environment a significant distance from the present setting. If so, heavy mineral assemblages represent original differences in sources.

Based on the known Juan de Fuca Plate motion and rate (Fig. 1), the early Pleistocene to late Pliocene age of sediments recovered at Sites 889 (Units II and III) and at Site 892 suggest the farthest paleogeographic positions at the time of deposition about $100 \mathrm{~km}$ to the southwest from the present locations of sites. The dispersion of data from Unit III at Site 888 (Fig. 3A) provides additional evidence for the migration of sediments from the southwest. This dispersion pos- sibly reflects the farthermost location of Unit III from the amphibolerich land masses of Vancouver Island and British Columbia in the past. It is unlikely that the dispersion of data reflects diagenesis or influence of wedge-derived fluids, because chemically unstable pyroxene is abundant. In particular, the relative enrichment of amphibole (Fig. 3) and magnetite (Table 1) among heavy minerals from Unit II at Site 888 supports the suggestion that Site 888 sediments migrated across the middle of the Nitinat Fan (Westbrook, Carson, Musgrave, et al., 1994), where the influence of drainages from Vancouver Island and British Columbia probably was strongest.

If the lower Pleistocene to upper Pliocene depocenters were located to the southwest, sediments recovered at Sites $889 / 890$ (Units II and III) were closer to the present location of Nitinat Fan during their deposition. It is logical to expect the increase of pyroxene and epidote and the possible relative decrease in amphibole in sediments from Unit III, as it was observed at Site 888 (Fig. 3A). The obtained data contradicts this suggestion. Pyroxene is relatively more abundant in sediments from Unit I, while amphibole is relatively abundant in the upper Pliocene sediments from Unit III (Fig. 3B).

The upper Pliocene sediments recovered at Site 892 have possibly formed closer to the Cascadia Range south or to the Klamath Mountains of southern Oregon and northern California. Sheidegger et al. (1973) have suggested that the provenance of the amphibole-dominated assemblage in the lower portion of the Pliocene sediments at DSDP Site 174A was the Klamath Mountains. The largely metamorphic terrane of the Klamath Mountains is known as a source of amphibole-rich and minor epidote detritus (Kulm et al., 1968; Sheidegger et al., 1971). This does not satisfactorily explain the significant increase in epidote and decrease in amphibole in a number of samples that we observed at Site 892 (Fig. 4B).

Westbrook (1994) calculated that for both accretionary wedges off Vancouver Island and Oregon, the accretion of a thick layer of sediment from the Cascadia Basin has occurred over the past few hundred thousand years and no more than about a million years. If so, the upper Pliocene sediments recovered at Sites $889 / 890$ and Site 892 had about 1 million years to travel on the plate, which restricts the migration distance to about $42 \mathrm{~km}$. The latter, in turn, restricts the probability of significant change in sources.

As we cannot find in the past adequate sources for the present day amphibole-epidote assemblage defined at Sites 889/890 and Site 892 and, thus, cannot explain the transition from the amphibole-pyroxene-epidote assemblage to the amphibole-epidote one by change in provenance, it is logical to assume that changes in mineral assemblages have been caused by processes acting within the sediments subsequent to their deposition.

The observed relative changes in mineral distribution may reflect sediment age only. Pyroxene and amphibole, which are not stable under burial conditions, were possibly dissolved in the lower Pleistocene to upper Pliocene sediments through diagenesis. At the same time, although sediments with the amphibole epidote-heavy mineral assemblage are older than those with the amphibole pyroxene epidote-heavy mineral assemblage, we cannot be sure that time alone is responsible for alteration and exclude possible tectonic or fluid influence on sediments, because of the complex postdepositional tectonics that probably have changed conditions of normal diagenesis.

The amphibole pyroxene epidote-assemblage was defined in sediments both unaffected (deep-sea fan and slope basins) and affected by accretionary tectonics (sediments from the toe of the accretionary wedge). If accretionary tectonics alone is responsible for heavy minerals alteration, then it is logical to find similar mineral assemblages at Sites 889/890 (Units II and III), at Site 891, and at Site 892. At the same time, we did not observe significant alteration at Site 891. It is possible, however, that the heavy mineral assemblage at Site 891 is not altered because it is located in the outer, most recently accreted part of the accretionary wedge, where neither time nor accretionary tectonics were capable of significantly modifying the heavy mineral 
assemblage. It is unlikely that accretionary tectonics alone have caused mineral alteration, as the degree of alteration in the sediments can be attributed largely to the presence of a liquid phase.

Active pore fluids, generated from tectonic overpressure, are well known in accretionary wedges and may cause dissolution of unstable minerals. The phenomenon of the intrastratal solution of certain minerals was observed in the upper Pleistocene to Pliocene sediments recovered in DSDP 18 (Scheidegger et al., 1973). It is likely that this phenomenon has occurred in Leg 146 Cascadia Margin sediments, since the heavy mineral assemblages are composed mostly of amphibole and pyroxene, which are unstable under burial conditions and are extremely sensitive to fluids. More stable garnet, staurolite, and sphene (as well as the minerals most resistant under burial conditions: zircon, tourmaline, and apatite) also demonstrate significant decreases in abundance within the inner parts of the accretionary wedge. The decrease of quartz amounts seems to be the best illustration of the intensity of fluid influence on sediments. Glauconite is also prominent in the light fraction. Most glauconite was found in the form of well-rounded pellets. However, a number of petrological features, listed in detail by Westbrook, Carson, Musgrave, et al. (1994), suggests authigenic formation of glauconite. The inverse relation of glauconite with quartz in the light fraction against the background of significant increase in pyrite and decrease in most heavy minerals in the heavy fraction provides evidence for its authigenic formation in situ.

It was also supposed (Scheidegger et al., 1973) that intrastratal mineral solution needs time to occur. We observed relative decrease in abundance of certain heavy minerals accompanied by an increase in pyroxene etching with depth and time at Sites 889/890 These trends are evident in Fig. 3B and, especially, at Fig.4B, that supports this position.

We registered changes in the relative abundance of certain heavy minerals at sites with anomalous fluid regimes and gas-hydrates occurrences, which seem to be important evidence of fluid influence on mineral assemblages. Differences in the heavy mineral assemblages at Sites 891 and lower units at Sites $889 / 890$ have most probably resulted from different fluid regimes. This suggestion correlates well with hydrological and geochemical data that indicate anomalous flows and with gas-hydrate occurrence at Site 892 (Westbrook, Carson, Musgrave, et al., 1994).

If fluid flow has affected the lower Pleistocene to upper Pliocene accreted sediments and fluid-related diagenesis is still occurring, it should be affecting the slope sediments as well as the accreted ones. Dispersion of data from Holocene to early Pleistocene Unit I at Sites $889 / 890$ (Fig 4B) seems to reflect the influence of fluid-related diagenesis on both accreted and slope sediments.

Finally, it is logical to conclude that both time and chemically active fluids are most responsible for heavy minerals alteration. Changes in relative abundance of certain heavy minerals have most probably resulted from strengthening normal diagenesis by active fluids generated from to tectonic overpressure in the accretionary wedge. Postdepositional alteration of heavy mineral assemblages provides problems in determining actual provenance of a deposit, even in relatively modern sediments. The possibility of intrastratal solution especially must be taken into consideration during reconstruction of provenance of ancient accretionary wedge fragments exposed on land in the present-day structure in numerous accreted terrianes.

\section{ACKNOWLEDGMENTS}

We wish to thank the American National Science Foundation, which supported the membership of one of the authors in Leg 146, and the Russian Foundation for Fundamental Research (grant No. 9305-9370) for financial support in the present study. We wish to thank Dr. R.V. Ingersoll and Prof. G.G. Zuffa for reviewing the manuscript and for making suggestions for its improvement.

\section{REFERENCES}

Carlson, P.R., and Nelson, C.H., 1987. Marine geology and resource potential of Cascadia Basin. In Scholl, D.W., Grantz, A., and Vedder, J.G. (Eds.), Geology and Resource Potential of the Continental Margin of Western North America and Adjacent Ocean Basins-Beaufort Sea to Baja California. Circum.-Pac. Counc. Energy Miner. Res., Earth Sci. Ser., 6:523-535.

Carson, B., 1971. Stratigraphy and depositional history of Quaternary sediments in Northern Cascadia Basin and Juan de Fuca Abyssal plain, northeast Pacific Ocean [Ph.D. dissert.]. Univ. Washington, Seattle.

Demets, C., Gordon, R.G., Argus, D.F., and Stein, S., 1990. Current plate motions. Geophys. J. Int., 101:425-478,

Duncan, J.R., and Kulm, L.D., 1970. Mineralogy, provenance, and dispersal history of late Quaternary deep-sea sands in Cascadia Basin and Blanko Fracture zone off Oregon. J. Sediment. Petrol., 40:874.

Duncan, R.A., and Kulm, L.D., 1989. Plate tectonic evolution of the Cascades arc-subduction complex. In Winterer, E.L., Hussong, D.M., and Decker, R.W. (Eds.), The Eastern Pacific Ocean and Hawaii. Geol. Soc. Am., 413-438.

Easterbrook, D.J., 1963. Late Pleistocene glacial events and relative sea level changes in the northern Puget Lowland, Washington. Geol. Soc. Am. Bull., 74:1465.

Hyndman, R.D., 1988. Dipping seismic reflectors and electrically conductive zones, metamorphic reactions and free water beneath a subduction zone. J. Geophys. Res., 93:13391-13405.

Hyndman, R.D., Spence, G.D., Yuan, T., and Davis, E.E., 1994. Regional geophysics and structural framework of the Vancouver Island margin accretionary prism. In Westbrook, G.K., Carson, B., Musgrave, R.J., et al., Proc. ODP, Init. Repts., 146 (Pt. 1): College Station, TX (Ocean Drilling Program), 399-419.

Kulm, L.D., Scheidegger, K.F., Byrne, J.V., and Spigai, J.J., 1968. A preliminary investigation of the heavy mineral suites of the coastal rivers and beaches of Oregon and Northern California. Ore Bin., 30:165-180.

Kulm, L.D., von Huene, R., et al., 1973. Init. Repts. DSDP, 18: Washington (U.S. Govt. Printing Office).

Nelson, C.H., Carlson, P.R., Byrne, J.V., and Alpha, T.R., 1970. Development of the Astoria Canyon-fan physiography and comparison with similar systems. Mar. Geol., 8:259-291.

Riddihough, R.P., 1984. Recent movements of the Juan de Fuca plate system. J. Geophys. Res., 89:6980-6994.

Scheidegger, K.F., Kulm, L.D., and Piper, D.J.W., 1973. Heavy mineralogy of unconsolidated sands in northeastern Pacific sediments: Leg 18, Deep Sea Drilling Project. In Kulm, L.D., von Huene, R., et al., Init. Repts. DSDP, 18: Washington (U.S. Govt. Printing Office), 877-888.

Scheidegger, K.F., Kulm L.D., and Runge, E.J., 1971. Sediment sources and dispersal patterns of Oregon continental shelf sands. J. Sediment. Petrol., 41:1121-1120.

Westbrook, G.K., 1994. Growth of accretionary wedges off Vancouver Island and Oregon. In Westbrook, G.K., Carson, B., Musgrave, R.J., et al., Proc. ODP, Init. Repts., 146 (Pt. 1): College Station, TX (Ocean Drilling Program), 381-388.

Westbrook, G.K., Carson, B., Musgrave, R.J., et al., 1994. Proc. ODP, Init. Repts., 146 (Pt. 1): College Station, TX (Ocean Drilling Program).

Whetten J.T., Kelley, J.C., and Hanson, L., 1969. Characteristics of Columbia River sediment transport. J. Sediment. Petrol., 39:1149.

Wiese, W., 1969. Studies in properties, distribution, and heavy mineral contents of sediments in northern Queen Charlotte Sound [B.Sc. thesis]. Univ. British Columbia, Vancouver.

\author{
Date of initial receipt: 12 September 1994 \\ Date of acceptance: 29 March 1995 \\ Ms 146SR-203
}

